\title{
Feasibility Study for the Development of a Surface Plasmon Resonance spectroscopy - Based Sensor for the BNFL-Hanford
}

by

B. B. Anderson

Westinghouse Savannah River Company

Savannah River Site

Aiken, South Carolina 29808

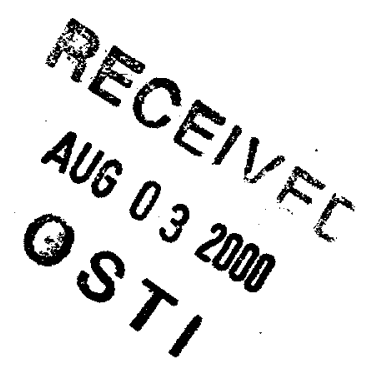

K. R. Powell

S. M. Serkiz

DOE Contract No. DE-AC09-96SR18500

This paper was prepared in connection with work done under the above contract number with the U.S. Department of Energy. By acceptance of this paper, the publisher and/or recipient acknowledges the U.S. Government's right to retain a nonexclusive, royalty-free license in and to any copyright covering this paper, along with the right to reproduce and to authorize others to reproduce all or part of the copyrighted paper. 


\section{DISCLAIMER}

This report was prepared as an account of work sponsored by an agency of the United States Government. Neither the United States Government nor any agency thereof, nor any of their employees, makes any warranty, express or implied, or assumes any legal liability or responsibility for the accuracy, completeness, or usefulness of any information, apparatus, product or process disclosed, or represents that its use would not infringe privately owned rights. Reference herein to any specific commercial product, process or service by trade name, trademark, manufacturer, or otherwise does not necessarily constitute or imply its endorsement, recommendation, or favoring by the United States Government or any agency thereof. The views and opinions of authors expressed herein do not necessarily state or reflect those of the United States Government or any agency thereof.

This report has been reproduced directly from the best available copy.

Available for sale to the public, in paper, from: U.S. Department of Commerce, National Technical Information Service, 5285 Port Royal Road, Springfield, VA 22161,

phone: (800) 553-6847,

fax: (703) 605-6900

email: orders@ntis.fedworld.gov

online ordering: http://www.ntis.gov/ordering.htm

Available electronically at http://www.doe.gov/bridge

Available for a processing fee to U.S. Department of Energy and its contractors, in paper, from:

U.S. Department of Energy, Office of Scientific and Technical Information, P.O. Box 62,

Oak Ridge, TN 37831-0062,

phone: (865)576-8401,

fax: (865)576-5728

email: reportsladonis.osti.gov 


\section{DISCLAIMER}

Portions of this document may be illegible in electronic image products. Images are produced from the best available original document. 
June 30,2000

BNF-003-98-0308

\title{
Feasibility Study for the Development of a Surface Plasmon Resonance Spectroscopy-Based Sensor for the BNFL-Hanford Technetium Monitoring Program
}

\author{
Brian B. Anderson, Kimberly R. Powell, and Steven M. Serkiz \\ Westinghouse Savannah River Co., Building 773-A, Aiken, SC \\ 29808
}

\section{DISCLAIMER NOTICE}

This report was prepared by Westinghouse Savannah River Company, Inc. (WSRC) on behalf of the U.S. Department of Energy (DOE) as an account of work sponsored by BNFL, Inc. Neither WSRC, nor DOE, the U.S. Government, or any person acting on their behalf, makes any warranty, expressed or implied, or assumes any legal liability or responsibility for the accuracy, completeness, or usefulness of any information, apparatus, product, or process disclosed, or represents that its use would not infringe privately owned rights. Reference herein to any specific commercial product, process, or service by trade name, trademark, manufacturer, or otherwise, does not necessarily constitute or imply its endorsement, recommendation, or favoring by WSRC, DOE, or the U.S. Government. The views and opinions of the authors expressed herein do not necessarily state or reflect those of WSRC, DOE, or the U.S. Government

Savannah River Technology Center Westinghouse Savannah River Company Aiken, SC 29808 
BNF-003-98-0308

June 30, 2000

Page ii of 30

Approvals

$\frac{\text { Buaserae }}{\text { Brian B. Anderson }} \frac{7-21-2000}{\text { Date }}$
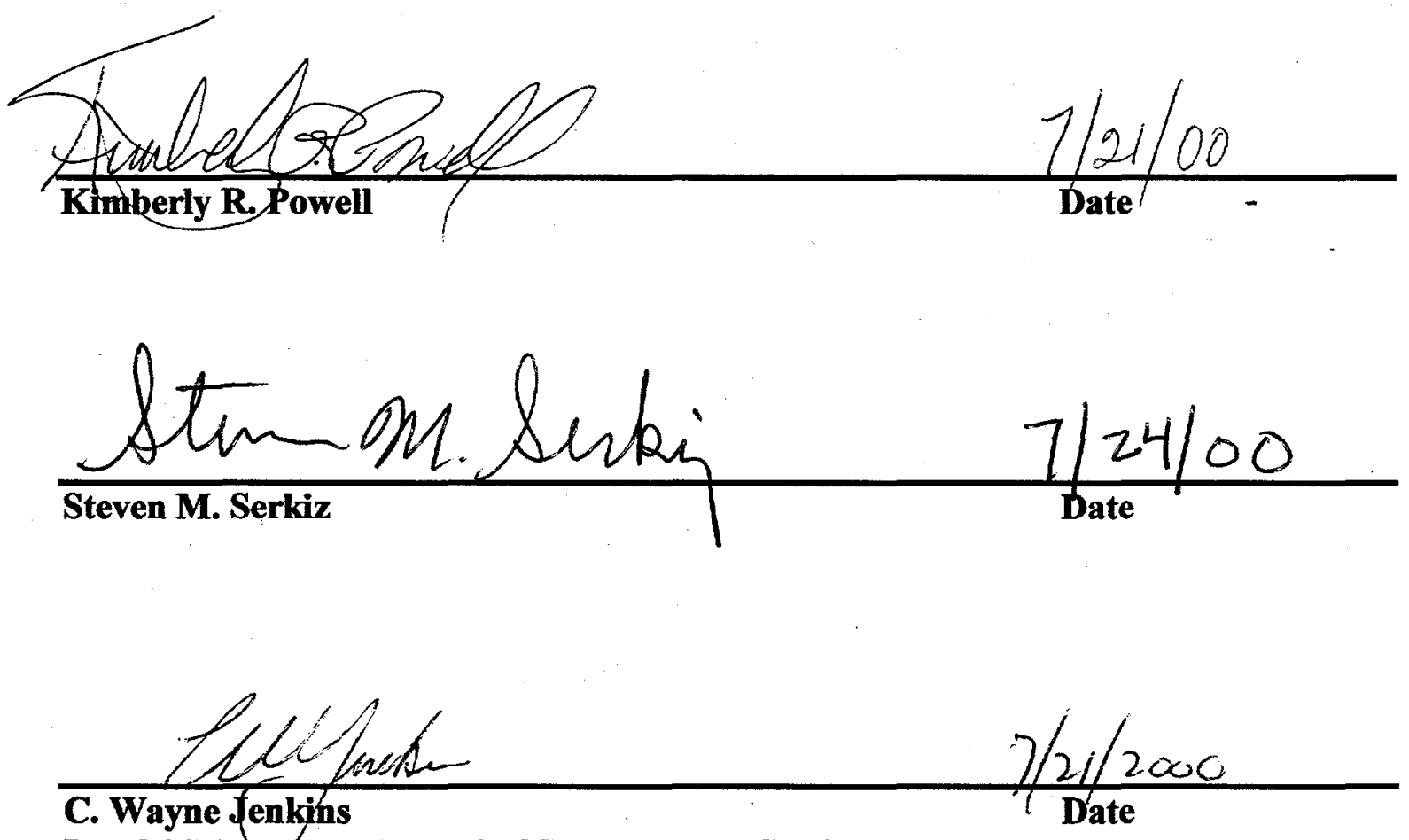

Level 3 Manager - Analytical Development Section 


\section{Table of Contents}

$\begin{array}{ll}\text { SubStrate Preparation } & 7\end{array}$

Poly(Ethylene Glycol) Self Assembled Monolayer (SAM) Preparation 8

$\begin{array}{ll}\text { SPECTROSCOPIC ELLIPSOMETRY } & 8\end{array}$

$\begin{array}{ll}\text { EXPERIMENTAL APPARATUS AND SPR SENSOR CHARACTERIZATION } & 8\end{array}$

RESULTS AND DISCUSSION

POLY(ETHYLENE GLYCOL) FILM Formation ON GOLd COATED Silicon WaFERS 11

$\begin{array}{ll}\text { Surface Plasmon Resonance Sensor Performance } & 14\end{array}$

IN-Situ POLy(ETHYLENE GLYCOL) FILM FORMATION 19

PEG Functionalized SPR SENSOR RESPONSE TO PERRHENATE $\quad 23$

$\begin{array}{lrl}\text { CONCLUSIONS } & 26\end{array}$

$\begin{array}{lr}\text { REFERENCES } & \mathbf{2 8}\end{array}$ 


\section{Table of Figures}

Figure 1. Optical ray-trace of the SPR probe fabricated using a commercially available attenuated total reflection spectroscopy probe.

Figure 2. SPR reflectivity for a three layer structure which would form basis for pertechnetate sensor

Figure 3. Poly(ethylene glycol) thiol attaches to gold via the thiol group and forms a thiolate with the gold.

Figure 4. Comparison between experimental SPR spectrum (above) and theoretical spectrum (below) shows that the SRTC fiber optic SPR probe generates high quality, high resolution spectra.

Figure 5. SPR sensor response to a series of sodium chloride solutions shows the low noise, high sensitivity response of he SRTC SPR Probe.

Figure 6. SPR sensor refractive index calibration results show the nonlinear relationship between the SPR wavelength and refractive index of the sample.

Figure 7. SPR sensor temperature stabilized response to circulated deionized water shows excellent stability for short term experiments and provides a baseline for determining the minimum detectable change in bulk refractive index

Figure 8. SPR sensor response to sequential flow stoppage

Figure 9. SPR sensor response to adsorption of monomethylated PEGthiol (M.W. $=5000$ ) (above) and temperature fluctuation during the experiment (below).

Figure 10. First hour of PEG adlayer formation (above), as shown in figure 9 , where the temperature fluctuation is shown (below).

Figure 11. SPR sensor response to $4 \mathrm{M} \mathrm{NaOH}$ blank and $4 \mathrm{M} \mathrm{NaOH}$ with 1 and $10 \mathrm{ppm}$ perrhenate. 23

Figure 12. Full sequence of perrhenate exposures shows the difference in response between the blank and the $10 \mathrm{ppm}$ perrhenate solutions. 
BNF-003-98-0308

June 30, 2000

Page $v$ of 30

\section{Table of Tables}

Table 1. Standard salt solutions used in characterization of the SPR sensor to refractive index perturbations.

Table 2. Refractive index and thickness measurement results for Si/gold \#1 wafer, successive washes after formation of PEG adlayer. 


\section{Abstract}

This report is the deliverable for a task assigned to the Sensors and Analyzers Technology group for the development and feasibility demonstration of a liquid phase pertechnetate sensor based on surface plasmon resonance. SRTC has developed a novel fiber optic SPR sensor for gas phase dewpoint determination, and we applied this same SPR probe structure, after suitable material modification, to liquid phase sensing. A literature search was conducted regarding the surface adsorption properties of pertechnetate ion with aim of identifying candidate materials for coating an SPR probe. Poly(ethylene glycol), which has been used in aqueous biphasic extraction chromatography for removal of pertechnetate from Hanford and Oak Ridge tank waste, was chosen as the sensor coating. We fabricated a prototype SPR sensor system consisting of a sapphire hemisphere coated with a thin gold film and then subsequently coated with PEG in-situ via the formation of self assembled monolayers (SAMs) of PEG attached to the gold surface via a thiolate bond. PEG SAMs were characterized by both SPR and spectroscopic ellipsometry and the PEG formation results compared favorably with literature reports of PEG monolayer formation on gold. The SPR sensor generated high resolution, low noise SPR spectra suitable for high sensitivity sensing of analyte adsorption onto the sensor surface. The fiber optic SPR sensor was able to discriminate changes in refractive index as small as $9.9 \times 10^{-7}$ RIU and exhibited a refractive index sensitivity of $1010 \mathrm{~nm} / \mathrm{RIU}$. We exposed the functionalized SPR probe to perrhenate solutions in $4 \mathrm{M} \mathrm{NaOH}$ and the sensor showed a unique response to $10 \mathrm{ppm}$ perrhenate. We believe this work demonstrates that the development of an SPR pertechnetate sensor is feasible. 


\section{Introduction}

The Department of Energy must treat and dispose of large volumes of radioactive waste stored in underground storage tanks at 5 DOE sites. Technology development has been focused on the separation and removal of various radionuclides from the supernatant contained in the Hanford waste tanks. The supernate consists of concentrated alkaline solutions of sodium nitrate and sodium nitrite with smaller amounts of hydroxide, aluminum, potassium, carbonate, sulfate, and phosphate. The primary sources of radioactivity in the supernate are Cs-137 and Tc-99. Tc-99 is a pure beta emitter with a half life of $2.13 \times 10^{5}$ years. It has been estimated that the Tc-99 activity in the supernate is on the order of $100-200 \mu \mathrm{C} / \mathrm{L}^{1}$. Technetium may form compounds with $\mathrm{Tc}$ oxidation states ranging from -1 to $+7^{2}$. The most predominant form of Tc-99 is Tc(VII) in the form of pertechnetate ion, $\mathrm{TcO}_{4}^{-}$. The water solubility and environmental mobility of pertechnetate ion require that it be removed from low-level waste streams.

The pertechnetate ion is the most stable form of Tc-99 in aqueous solutions, and Tc99 in oxidation states lower than +4 is stable in coordination complexes. There is evidence that up to $70 \%$ of the Hanford tank waste may be in forms other than pertechnetate and it has been hypothesized that pertechnetate is reduced in solution by the presence of organic complexants [ref. 10]. Lower oxidation state Tc-99 may be oxidized to pertechnetate prior to removal by anion exchange, and current research is focused on the effects of oxidizers on the extraction medium. The use of ion exchange columns for Tc-99 removal is the baseline technology, and the development of analyzers which detect Tc-99, or pertechnetate, breakthrough would aid in process control and optimization. An in-situ probe, which would measure pertechnetate ion by selective binding, would yield real-time data on the column performance and serve as an early detection device for column breakthrough without costly sampling or engineering of a side stream for at-line analysis.

We proposed the development of a selective, liquid phase surface plasmon resonance probe for technetium-99 in the pertechnetate form based on the functionalization of a novel surface plasmon resonance (SPR) spectroscopy probe developed by SRTC. This report details the literature survey results, proposed research path, and the results of the development of a poly(ethylene glycol) functionalized SPR sensor for detection of pertechnetate. The goal of this research is to determine SPR pertechnetate sensor feasibility based on literature survey and prototype development and testing.

\section{Surface Plasmon Resonance Spectroscopy}

The area of surface plasmon resonance sensors has received much attention in the literature, and recently an entire journal issue was devoted to SPR sensor development. ${ }^{3}$ A significant majority of the literature deals with liquid phase sensors, and a recent review covered the salient features of SPR sensor systems and reported applications. ${ }^{4}$ 
Surface plasmon resonance spectroscopy (SPR) measurements are made using optical systems which control parameters to which SPR is sensitive: incident angle; wavelength of incident light; degree of polarization; and the optical properties of the materials making up the sensor. In general, a typical SPR experiment requires a dielectric substrate (prism in most cases) which has been coated with precise thickness of a suitable noble metal. The combination of the substrate, noble metal, and sample in contact with the metal, allows for the generation and support of surface plasmon polaritons (SPPs) which are formed along the metal-dielectric interface. These polaritons are highly damped charge density waves oscillating at optical frequencies, and may be excited if the materials and optical properties of an experimental system are chosen correctly.

In general, the SPPs are not measured directly, but their presence is inferred by monitoring the light intensity reflected from the substrate/metal interface. The reflected light will contain a deep minimum in intensity, and this minimum occurs at what is termed the resonance for the surface polariton, or plasmon. The monitoring of the surface plasmon reflectivity curve, and in particular, the position of the resonance, yields what is generally known as surface plasmon resonance spectroscopy (SPRS). The position of the reflectivity minimum is very sensitive to the interface properties between the prism face and the liquid. Changes in metal film thickness, surface adsorbed species, and liquid dielectric properties are all seen as changes in the SPR resonance wavelength. SPR has been shown to be sensitive to very low levels of material adsorbed onto a surface (much less than a monolayer) and has been used to characterize ultra-thin films ${ }^{5,6}$.

SPRS is used to determine the optical properties of the materials which make up the structure which supports SPR, and this structure includes the metal layer and anything in contact with the metal layer. The plasmon wave decays exponentially from the surface of the metal and penetrates into the sample approximately $200-300 \mathrm{~nm}$, depending upon the materials. Different configurations have been employed with the goal of miniaturization. Significant effort has been made towards the development of compact SPR systems and probe geometries that enable the collection of research or bench top quality SPR spectra from fiber optic-based sensors. Several fiber optic based sensors have been developed for liquid phase bio-assay, most notably the multi-mode fiber based SPR probe marketed by Pharmacia ${ }^{7,8}$. A single-mode optical fiber SPR sensor system was described by Slavík, et al. for application in liquid systems. ${ }^{9}$ A fiber coupled compact planar surface plasmon resonance transducer, which makes use of a faceted probe end and miniature coupling optics, was described and applied in bio-sensing experiments ${ }^{10}$.

SRTC has developed a novel SPR probe which enables the acquisition of high quality, high resolution SPR spectra using a simple, robust fiber optic probe consisting of a single bulk optical element with no facets. The SPR probe is based on a commercially available hemispherical attenuated total reflection spectroscopy probe (Equitech, International). The probe consists of a stainless steel 0.5 inch diameter housing which contains optical fibers which are polished and spaced appropriately at the distal end. The fibers are arranged such that the launch and collection fibers are placed at the focal points for propagation through the ATR hemisphere. The ATR probe design, by Equitech, takes advantage of the geometrical optics associated with internal reflections along the hemisphere, where the rays are traced along inscribed polygons $(4,5,6$ sided, etc.), to 
BNF-003-98-0308

June 30, 2000

Page 3 of 30

SPR is supported on the apex by application of thin gold film

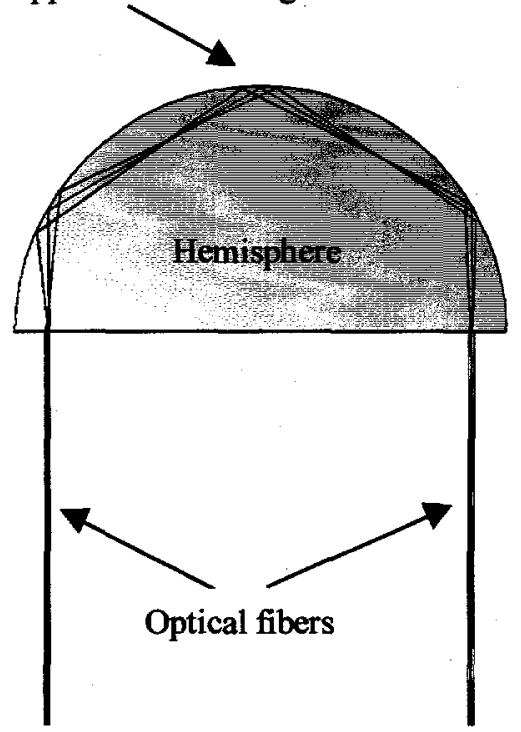

Figure 1. Optical ray-trace of the SPR probe fabricated using a commercially available attenuated total reflection spectroscopy probe. Optical fibers deliver the incident light to the flat face of the hemisphere and then collect the reflected light. The incident angle is held constant at $60^{\circ}$ by the curvature of the optic for this three bounce probe. This structure represents the first monolithic, high resolution fiber optic SPR probe. perform ATR measurements without relying on a faceted crystal. Figure 1 shows the configuration used in these SPR experiments, where the light is incident upon the hemisphere in an ATR configuration. This SPR probe allows SPR excitation at any or all of the internal reflection points along the surface without the need for external focusing or collimating optics. The monolithic construction (single optical element) and incident angle purity achieved by the hemisphere curvature yields a fiber optic coupled probe which generates high quality SPR spectra. Previously reported SPR probe configurations require additional optical components and facets to generate similar quality SPR spectra. In our own applications we have shown the technique to be sensitive to sub ppm levels of moisture in air.

An approach to the development of an in-situ pertechnetate sensor would be to take advantage of the SPR probe surface sensitivity and the pertechnetate ion's unique sorption properties on certain metals. Pertechnetate has been shown to be a strong corrosion inhibitor for steel and iron, and this inhibitor role is due to the strong adsorption of pertechnetate ion on metal surfaces. A fundamental study from 1953 was found which described the interactions of pertechnetate with ferrous metals for corrosion inhibition applications ${ }^{11}$. Pertechnetate ion exhibited corrosion inhibition with solution concentrations down to $2-5 \mathrm{ppm}$ for iron and metals containing ferrous material under neutral to basic conditions. This study formed the basis for

undertaking the literature survey with the goal of determining the feasibility of applying SPR to pertechnetate analysis by way of metal/ion interactions.

\section{Literature Search Results: Feasibility of Sensor Development}

A search was undertaken using SciFinder ${ }^{\mathrm{TM}}$ and the Ei Tech Index. The SciFinder ${ }^{\mathrm{TM}}$ search using the term (technetium or pertechnetate) yielded 1786 hits. Refining the search by adding the term "metal" yielded 198 hits. Analysis of older references (19661986) resulted in 23 references. One reference, again by Cartledge, further discussed the mechanism for corrosion inhibition on iron and steel surfaces ${ }^{12}$. The other references discussed, for the most part, technetium complexes and chelating agents for biomedical applications and recovery of technetium from nuclear wastes. Of the 198 references found in the refined search, only 7 contained the word surface, and only one, reference 2 in the endnotes, discussed surface activity of pertechnetate. A refinement of the initial search to include the concept "adsorption or adsorb" yielded $73 \mathrm{hits}$, and one reference to 
the adsorption of Tc-99m ( 6 hour half-life, $142.7 \mathrm{KeV}$ gamma, used as a short lived tracer) on silver coated activated carbon at low $\mathrm{pH}^{13}$. An additional reference dealing with corrosion inhibition was also found ${ }^{14}$. Very little in addition to the referenced work on corrosion inhibition has been published regarding the surface activity of pertechnetate ion on metal surfaces. It is clear that if a sensor is designed based on a corrosion inhibition property of pertechnetate, additional fundamental work in the surface chemistry of pertechnetate will need to be performed. It is unclear as to whether the noble metals required by SPR spectroscopy constraints will have sufficient affinity for pertechnetate, and additionally it is not likely that the response will be entirely selective for pertechnetate. Waste streams will most likely have varying salt and alkaline conditions based on waste tank source, and a sensor design will have to incorporate a technique for distinguishing non-specific binding events. Based on the lack of literature resources on the subject of adsorption of pertechnetate ion on noble metal surfaces, this line of development may be time and cost prohibitive.

Another approach to sensor development is the application of a thin, chemically selective film onto the SPR probe which would facilitate the sensing through chemisorption or partitioning of pertechnetate ion. Several reports have been issued in the DOE Complex dealing with the interactions of pertechnetate ion with potential removal technologies. Bartsch ${ }^{15}$ worked from 1988-1994 in the design, synthesis, and characterization of ionizable crown ether based ligands for use as chelating agents in solvent extraction and liquid membrane transport separation chemistries. The focus of the research was on designing chelating agents for mono- and divalent metal cations. In addition, polycyclic ether functionalized exchange resins were prepared for evaluation as solid phase extraction media. These resins were formed by condensation polymerization to form weak acid exchange resins. He demonstrated that the conformation of the resin functional groups affect the binding and selectivity of the resin. This indicates that resins may be prepared which have a certain surface conformation which makes them selective for a particular analyte. However, the review did not discuss using the crown ether extractants on negatively charged species.

A report by Blanchard, et $\mathbf{a l}^{16}$ details the removal and characterization technologies which have been evaluated for treatment of Hanford Tc bearing waste. Two separation media, Reillex ${ }^{\mathrm{TM}}$ HPQ (Reilly Industries) and ABEC 5000 (Eichrom Industries), were evaluated for their removal properties for two types of waste. Much work has focused on the Reillex ${ }^{\mathrm{TM}}$-HPQ resin. Schroeder, et al $^{17}$ reported the use of Reillex ${ }^{\mathrm{TM}}$-HPQ for pertechnetate removal from caustic solutions similar to waste tank environments. Reillex ${ }^{\mathrm{TM}}-\mathrm{HPQ}$ is a modified copolymer of divinylbenzene and 4-vinylpyridine. The unique characteristics of the resin arise from the methylated pyridine functionality which imparts strong base anionic sites to the copolymer. This structure is more stable than other resins with respect to radiolysis and nitric acid degradation. Technetium, in the form of pertechnetate, partitioned into the resin with distribution coefficients, $K_{d}$, between 200 and 550. The hydroxide form of the resin was used for breakthrough studies due to gas formation in the resin bed when the Hanford simulant is processed with the nitrate form. Strong base anion exchange resins swell when converted from the nitrate to hydroxide form. Film swelling will be an issue for any thin film sensor development program, and steps will need to be taken to minimize or correct for film changes due to 
BNF-003-98-0308

June 30, 2000

Page 5 of 30

changes in solution character. Schroeder also noted that chromium in the form of chromate is not sorbed by the resin. This indicates that the resin has a high specificity for pertechnetate over chromate, and non-specific binding in a sensor configuration is expected to be minimized. Schroeder continued work on the elucidation of the partitioning characteristics of the pertechnetate/Reillex ${ }^{\text {TM}}$-HPQ system under varying ionic strengths and caustic conditions ${ }^{18}$. They applied the use of a Tc99m tracer, which is a gamma emitting medical isotope of Tc99, and this allowed more precise determination of $\mathrm{Kd}$ values and decreased counting times. At high nitrate $(>1 \mathrm{M})$ concentration, the effects of competitive nitrate adsorption dominate and the $\mathrm{Kd}$ for pertechnetate becomes a function of nitrate concentration and independent of hydroxide at lower hydroxide concentrations. Above $1 \mathrm{M}$ hydroxide, the resin becomes dehydrated to some extent and the affinity for pertechnetate over nitrate increases, indicating better performance of the resin for higher hydroxide concentrations. The work performed in these studies indicate that the Reillex ${ }^{\mathrm{TM}}$-HPQ resin may be useful as a sensor surface, provided the non-specific binding events be minimized or accounted for in the signal processing.

Another sorbent material used in pertechnetate removal from Hanford wastes is the ABEC-5000 resin, based on the aqueous biphasic system (ABS). A water soluble polymer, polyethylene-glycol (PEG) is used as a sorbent material. At high salt concentrations, the solution will separate into two aqueous phases, a PEG-rich phase and a high salt phase. The partitioning behavior of this material with respect to Hanford waste streams has been studied by Rogers, et al $^{19}$, and they found that large, poorly hydrated anions, such as pertechnetate, partitioned quantitatively into the PEG-rich phase of an ABS. The advantage of an ABS based separation is that the elution may be carried out in deionized water, and this would simplify sensor regeneration. In contrast, regeneration of anion exchange resins require addition of $8 \mathrm{M}$ nitric acid or reduction and complexation by addition of stannous ion and ethylenediamine. Rogers, et $\mathrm{al}^{20}$ also evaluated the performance of a resin used in aqueous biphasic extraction chromatography, where monomethylated PEG-5000 was immobilized onto a polystyrene support and tested in batch mode under varying ionic strengths and ionic species. They found that the $\mathrm{ABEC}-5000$ resin effectively removed pertechnetate from simulated waste tank samples. A study at Oak Ridge National Laboratory showed that ABEC-5000 achieved higher distribution coefficients than Reillex ${ }^{\mathrm{TM}}-\mathrm{HPQ}$ resin for pertechnetate removal in Melton Valley Storage Tank Samples ${ }^{21}$. However, tests using Hanford waste samples resulted in distribution coefficients an order of magnitude below those reported in the ORNL study ${ }^{22}$.

A new technology for pertechnetate extraction involves the use of an immobilized crown ether extractant from IBC Industries known as Superlig-639 and this material has been selected as the resin for Hanford waste treatment. Superlig-639 is based on a polystyrene backbone which has been functionalized with a crown ether ligand at the correct conformation. The structure and synthesis are proprietary, but there has been some work in liquid phase solvent extraction using crown ethers. Reference 15 discussed the synthesis of a wide variety of crown ethers specifically for metal ion complexation. However, any development of sensors based on this material must be undertaken jointly with IBC Industries. 


\section{Initial Research Plan for SPR Sensor Development}

The SPR technique is used for immunoassays and to monitor binding events in biologically pertinent reactions. In standard laboratory experiments, SPR can monitor specific binding events with reportable mass detection limits of 10 to $15 \mathrm{pg} / \mathrm{mm}^{23,24}$. This sensitivity allows SPR to be used for affinity measurements under very low concentration conditions. Provided a suitable surface can be prepared with an affinity for pertechnetate, a column breakthrough sensor is entirely feasible based on this technology.

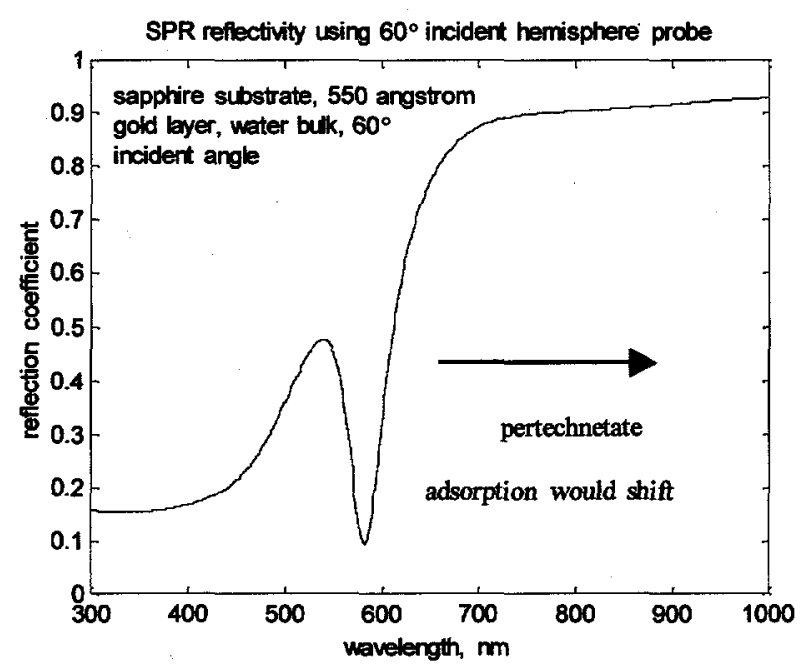

Figure 2. SPR reflectivity for a three layer structure which would form basis for pertechnetate sensor.
Figure 2 displays the theoretical SPR reflectivity curve for a structure that would be employed in pertechnetate sensing. The preferred format for pertechnetate sensing as it relates to column breakthrough would involve a sensor with a sorbent layer identical to the medium used for pertechnetate removal in the ion exchange process. However, demonstration of a sensor based on a non-ideal material would be of benefit in terms of feasibility determination. Therefore, the polyethethylene-glycol system was investigated initially with the goal of demonstrating pertechnetate response in a liquid phase SPR sensor. PEG is the least proprietary material in the set of evaluated technologies and has a significant research base from which to draw. The literature review indicates that the development of a sensor is feasible based on a pertechnetate selective sorbent layer coupled with SPR detection.

The remainder of the report will detail the development of a prototype liquid phase SPR system suitable for investigating the proposed sensor response. We chose nonradioactive rhenium, which forms the perrhenate ion in solution and has been used in other partitioning studies, as a surrogate for pertechnetate ${ }^{15}$. The milestones for sensor development set forth following the literature review are as follows:

1. Fabricate a liquid SPR sensor based on the materials and parameters given in figure 2 (sapphire hemisphere with $550 \AA$ gold layer).

2. Test the sensitivity of the probe with various salt solutions to determine refractive index response.

3. Investigate the film formation of PEG polymer onto a suitably modified gold surface using spectroscopic ellipsometry. 
4. Design, fabricate, and characterize an SPR flow system.

5. Investigate PEG film formation by SPR in-situ.

6. Prepare a perrhenate sensor based on PEG film and evaluate response to perrhenate under Hanford simulant conditions.

Successful completion of the objectives will provide an indication of the viability of the sensor platform for process sensing as well as yield valuable information as to the feasibility of the proposed sensing scheme. If the sensor responds to perrhenate with some degree of confidence, future work should be initiated in the preparation of additional sensors which may be tested in pertechnetate solutions in neat and Hanford waste simulant environments.

\section{Experimental Section}

\section{Materials}

A stainless steel attenuated total reflection probe and sapphire hemispheres were purchased from Equitech International. A chromium metal sputtering target (Kurt J Lesker Co.) and gold foil (99.9\% purity) were used in the fabrication for the SPR sensor elements. One inch diameter doubly polished silicon wafers (Silicon Quest International) were used for ellipsometry studies. Deionized water (Barnstead, 17.6 MOhm-cm), solid sodium chloride, sodium hydroxide pellets, methanol, and concentrated nitric acid (all Fisher, ACS reagent grade) were used to clean substrates and prepare solutions for SPR sensor characterization. Poly(ethylene glycol) (monomethylated thiol), molecular weight 5,000 , was obtained from Shearwater Polymers and stored at $-10^{\circ} \mathrm{C}$ prior to use. Upon removal of material from the bottles, each was returned to low temperature storage. $\mathrm{HNO}_{3}(2 \%)$ was prepared from distilled concentrated $\mathrm{HNO}_{3}$ (Optima grade, Fisher Scientific) by dilution into deionized water. Rhenium standards were prepared from $1000 \mathrm{mg} / \mathrm{L}$ Re standard (High Purity Standards, Charleston, SC) which were diluted into $4 \mathrm{M}$ sodium hydroxide in order to simulate the high ionic strength and basic conditions of tank waste without the hazards associated with radioactive materials.

\section{Substrate Preparation}

Sapphire hemispheres and silicon wafers were cleaned with methanol and deionized water and dried in an argon jet. A dual target DC planar magnetron (base pressure $<1 \times 10^{-7}$ torr) was used to sputter coat masked alumina hemisphere substrates with a flash layer of chromium $(\sim 20 \AA$, sputtering conditions: 5.0 mtorr Ar, 25 watts $)$ and 500-550 $\AA$ gold (5.0 mtorr Ar, 25 watts, $3.1 \AA / \mathrm{s}$ ) layer. Silicon substrates were coated with approximately $1000 \AA$ gold in order to generate an optically thick film for ellipsometry. Thickness was monitored during deposition with a quartz crystal microbalance. 


\section{Poly(Ethylene Glycol) Self Assembled Monolayer (SAM) Preparation}

A representative SAM preparation with $5000 \mathrm{MW}$ PEG-SH in EtOH: $\mathrm{CH}_{2} \mathrm{Cl}_{2}$ is as follows: gold coated silicon wafers and solvent $(50 \mathrm{~mL}$ of anhydrous ethanol and $25 \mathrm{~mL}$ of methylene chloride) were sparged with nitrogen for $0.5 \mathrm{~h}$ in a custom designed glass apparatus with a large o-ring seal to accommodate large substrates under inert atmosphere. The $5000 \mathrm{MW}$ methoxypoly(ethylene glycol)-thiol, PEG-SH, $\left[\mathrm{CH}_{3} \mathrm{O}\left(\mathrm{CH}_{2} \mathrm{CH}_{2} \mathrm{O}\right)_{\mathrm{n}} \mathrm{CH}_{2} \mathrm{CH}_{2} \mathrm{SH}, \mathrm{n} \approx 110\right] 92 \%$ with $3 \%$ PEG-disulfide present as an impurity was weighed in air and added as a solid under a stream of nitrogen to the 2:1 EtOH: $\mathrm{CH}_{2} \mathrm{Cl}_{2}$ solution. The substrates were allowed to stand for at least $10 \mathrm{~h}$ under nitrogen in the resulting $0.7 \mathrm{mM}$ PEG-SH solution. The vessel was then opened and the substrates removed and rinsed first with methylene chloride then ethanol repeating these rinses an additional two times followed by rinsing with deionized water. Substrates were then dried under nitrogen prior to ellipsometry measurements.

\section{Spectroscopic Ellipsometry}

Ellipsometry is useful in the determination of the optical constants and thicknesses of materials with angstrom resolution. It is routinely used in characterization of thin dielectric films deposited on silicon and metals, as well as the determination of the optical constants of optically thick metals. Surface or thin film optical parameters (refractive index and absorption) are calculated by fitting the experimental ellipsometric data (Psi and Delta) to physical models which describe the layer optical constants and thicknesses in general. The models may have multiple layers, and it is best to perform analyses on individual layers and then use the optical constants and thicknesses to build the model for entire stack. Spectroscopic ellipsometric characterization was performed on silicon wafers after each coating and functionalization step in order to determine the presence of each subsequent layer.

A J. A. Woollam M-44 ellipsometer with a fixed angle $\left(75^{\circ}\right)$ stage and Wvase 32 acquisition and analysis software was used in all characterization experiments under ambient laboratory conditions with the ellipsometric data taken in high resolution mode. Optical constants were determined for sputtered gold prior to functionalization and a Cauchy optical model was used to calculate thicknesses and optical constants of the resulting PEG self-assembled monolayers formed on the gold surfaces ${ }^{25}$.

\section{Experimental Apparatus and SPR Sensor Characterization}

The SPR probe is constructed from 0.5 inch stainless tubing and may be Swage-Loc fitted into a process stream. Therefore, a flow cell was constructed from Lexan which would accommodate the SPR sensor in a flowing stream contained in a 0.125 inch diameter flow channel. The cell also accepted a NIST-traceable platinum RTD temperature probe (Cole Parmer, $\pm 0.05^{\circ} \mathrm{C}$ ), which did not contact the fluid but rather measured the temperature of the block. The flow cell was constructed such that the apex of the SPR probe was placed in the center of the channel. The SPR probe was held in 
BNF-003-98-0308

June 30,2000

Page 9 of 30

place with a Teflon ferrule and Swage-Loc bushing. The flow cell was fitted to .125 inch interior diameter (id) Teflon tubing. The input line was connected to $0.5 \mathrm{~mm}$ id Teflon tubing and a peristaltic pump with a variable flow rate. The output line was directed to waste or to the sample bottle for recirculation. Samples were pumped continuously with pump stoppage during placement of new samples in the flow system. Flow rates were selected between .8 and $2.0 \mathrm{ml} / \mathrm{min}$. Most experiments were performed at $2.0 \mathrm{ml} / \mathrm{min}$, which corresponds to a linear flow velocity of $.42 \mathrm{~cm} / \mathrm{s}$ inside the .125 inch diameter flow channel, neglecting the intrusion of the probe tip into the middle of the channel. Static experiments were also conducted in the initial characterization of the SPR probe, where the probe was dipped into solutions of interest and then rinsed in deionized water between samples.

Table 1. Standard salt solutions used in Characterization of the SPR sensor to refractive index perturbations

\begin{tabular}{|c|c|c|c|c|}
\hline $\mathrm{NaCl}$ weight & $\begin{array}{c}\text { Total solution } \\
\text { weight }\end{array}$ & wt. $\% \mathrm{NaCl}$ & $\begin{array}{l}\text { Refractive index, } \\
20^{\circ} \mathrm{C} \text {, at sodium } \mathrm{d}- \\
\text { line }\end{array}$ & $\begin{array}{l}\text { Refiractive index at } \\
30^{\circ} \mathrm{C} \text {, sodium } \mathrm{d} \text {-line }\end{array}$ \\
\hline 0.0000 & 100.000 & $0.000 \%$ & 1.33291 & 1.33174 \\
\hline 0.5003 & 100.887 & $0.496 \%$ & 1.33379 & 1.33262 \\
\hline 0.9996 & 100.044 & $0.999 \%$ & 1.33468 & 1.33351 \\
\hline 2.0001 & 100.154 & $1.997 \%$ & 1.33646 & 1.33529 \\
\hline 3.0008 & 100.067 & $2.999 \%$ & 1.33823 & 1.33706 \\
\hline 3.9930 & 100.179 & $3.986 \%$ & 1.33998 & 1.33881 \\
\hline 5.0007 & 100.098 & $4.996 \%$ & 1.34178 & 1.34061 \\
\hline 6.0044 & 100.146 & $5.996 \%$ & 1.34355 & 1.34238 \\
\hline 7.0024 & 100.011 & $7.002 \%$ & 1.34534 & 1.34417 \\
\hline 8.0030 & 100.038 & $8.000 \%$ & 1.34711 & 1.34594 \\
\hline 9.0031 & 100.142 & $8.990 \%$ & 1.34887 & 1.34770 \\
\hline 10.0011 & 100.133 & $9.988 \%$ & 1.35064 & 1.34947 \\
\hline 12.0011 & 103.126 & $11.637 \%$ & 1.35356 & 1.35239 \\
\hline 14.0089 & 99.993 & $14.010 \%$ & 1.35777 & 1.35660 \\
\hline 16.0029 & 100.048 & $15.995 \%$ & 1.36130 & 1.36013 \\
\hline 18.0041 & 100.008 & $18.003 \%$ & 1.36486 & 1.36369 \\
\hline 20.0078 & 100.085 & $19.991 \%$ & 1.36839 & 1.36722 \\
\hline
\end{tabular}

The optical system consisted of the SPR probe (which contained UV grade 200 micron core diameter fibers for launch and collection of the light), light source, optical spectrometer and instrument controller. The SPR probe input fiber was coupled to a tungsten-halogen light source (Ocean Optics LS-1) while the collection fiber was inserted into the SMA input port of a Ziess MCS $501 \mathrm{UV}$-Vis spectrometer. The spectrometer optical bench was obtained commercially and mated to internally developed data acquisition and analysis hardware/software. The spectrometer has $0.8 \mathrm{~nm}$ pixel resolution with approximately $4 \mathrm{~nm}$ optical resolution with a wavelength range from 190 to $1100 \mathrm{~nm}$. Experiments were performed using a 15 scan signal average in absorbance mode such that the SPR reflectivity minimum appeared as a peak in the absorption spectrum when the system was referenced to air. The position of the surface plasmon resonance was calculated dynamically by applying a 5 point Guassian convolution smoothing filter and a least squares peak picking algorithm to the collected absorbance 
BNF-003-98-0308

June 30,2000

Page 10 of 30

spectra. Data were logged onto a Micron GoBook II laptop computer with the Windows 98 operating system. Experimental SPR spectra and a corresponding log file containing a time stamp and SPR peak position were saved onto the computer hard drive for later retrieval and analysis. Data processing and analysis was performed using Matlab ${ }^{\mathrm{TM}}$ (The Mathworks, Inc.) and Excel spreadsheet program (Microsoft).

The SPR sensor was characterized with respect to refractive index via a series of sodium chloride solutions prepared gravimetrically in deionized water. Table 1 lists the solutions and associated calculated refractive index values ${ }^{26}$ at $20^{\circ} \mathrm{C}$ and $30^{\circ} \mathrm{C}$. The values at $30^{\circ} \mathrm{C}$ were calculated using the dispersion of pure water. Two separate experiments were performed to compare the effects of dipping the probe versus flowing solutions over the probe tip in the flow cell. Samples were run randomly with several water blanks in order to test reproducibility and cell holdup.

The system was assembled and first tested under uncontrolled ambient conditions. It became apparent, however, that the temperature fluctuations in the laboratory were too great to allow for long term SPR experiments. Therefore, the probe, flow cell, temperature sensor, and liquid samples were placed into a laboratory incubator (Fisher) with $\pm 0.1^{\circ} \mathrm{C}$ stability. All temperature controlled experiments were performed at $30^{\circ} \mathrm{C}$. Solutions were allowed to temperature equilibrate for at least a day prior to each experiment, and fresh deionized water was stored in the incubator. The temperature control was not sufficient to allow heat sources to be placed into the incubator, so the light source, spectrometer, and peristaltic pump were placed in ambient. The peristaltic connections were extended and the time for sample to reach the probe was approximately 1 minute. Long term temperature stability in the incubator was determined to be $\pm 0.17^{\circ} \mathrm{C}$ over three days.

Poly(ethylene glycol) monolayers were formed in-situ by flowing PEG-SH solutions $(.05-1 \mathrm{mmol} / \mathrm{L})$ prepared in deionized water into the flow cell at a flow rate of 1 to 2 $\mathrm{ml} / \mathrm{min}$ and then allowing the solution to re-circulate for as much as 24 hours. Initial PEGylation of the SPR sensor gold surface was performed under ambient conditions (no temperature control) using a substrate that had been used in liquid systems for several months and subjected to sodium chloride calibration solutions. The sensor element was cleaned in ethanol, deionized water, and dried in a nitrogen stream. The element was replaced in the probe head and reference spectrum obtained in air after the probe had been inserted in to the flow cell and tightened. The sensor was then exposed to deionized water followed by $1 \mathrm{mmol} / \mathrm{L}$ PEG-SH solution, which was re-circulated overnight. Subsequent PEGylation of the SPR sensor occurred in the temperature controlled environment using either a $0.1 \mathrm{mmol} / \mathrm{L}$ or $0.05 \mathrm{mmol} / \mathrm{L}$ PEG-SH solution.

Perrhenate standards ( $1 \mathrm{ppm}$ and $10 \mathrm{ppm}$ ) were prepared in $4 \mathrm{M} \mathrm{NaOH}$ from 1000 ppm rhenium in $2 \%$ nitric acid. Additional $2 \%$ acid was added to the $\mathrm{NaOH}$ blank and the $1 \mathrm{ppm}$ perrhenate standard in order to match the composition of the $10 \mathrm{ppm}$ perrhenate standard. The SPR sensor was exposed to $\mathrm{NaOH}$ and perrhenate standards following the final PEG adlayer formation from $0.05 \mathrm{mmol} / \mathrm{L}$ PEG-SH on a hemisphere which was freshly coated with gold (placed into flow cell within 10 minutes of removal from the vacuum system). 


\section{Results and Discussion}

\section{Poly(ethylene glycol) Film Formation on Gold Coated Silicon Wafers}

For pertechnetate SPR sensor applications the high solubility of PEG in water necessitates some means of immobilization of this polymer on the gold surface of the SPR probe. There are a number of approaches to the PEGylation of surfaces reported in the literature including short chain $(\mathrm{MW}<1000)$ PEG-alkyl thiolate monolayers on gold and a number of routes for covalently bonding longer chain $(M W>1000)$ PEG to silica substrates. Most PEG functionalization of silica was performed in an attempt to passivate silica surfaces in electrophoresis applications where PEG oligomers and large polymers $(\mathrm{M} . \mathrm{W} .=25,000)$ were attached via a silica grafting procedure ${ }^{27}$. Short-chain alkylthiol monolayer formation has been studied with SPR where the adlayer thickness and optical properties were estimated from SPR data ${ }^{28}$. We were interested in attaching higher molecular weight PEG $(M W=5000)$ in order to mimic the ABEC 5000 resin which could be accomplished based on known technology by coating the gold surface of the hemisphere with an adlayer of silica followed by grafting of PEG to the silica. We preferred, however, functionalizing the gold surface using a thiol functionalized PEG to form a thiolate linkage for two reasons: the directness of the synthetic route and the more intimate contact between the PEG and the gold surface of the probe (see figure 3). Only one report was found, after work was initiated, which dealt with the attachment of large PEG molecules to gold. This route utilized an asymmetric orthopyridyl-disulfide functionalized PEG (OP-SS-PEG). ${ }^{29}$ Although 5000 MW PEG-thiol is commercially available, we found no reports of the functionalization of gold using this long chain PEGthiol. We believe this is the first attempt to functionalize a gold surface using a long chain PEG-thiol.

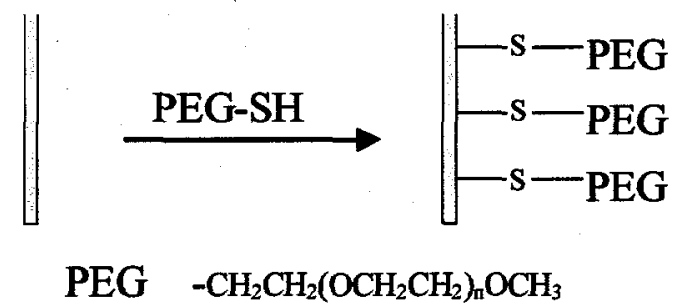

Figure 3. Poly(ethylene glycol) thiol attaches to gold via the thiol group and forms a thiolate with the gold. This reaction would yield a film of PEG which would serve as the aqueous biphasic extraction medium, where changes in the film due to absorption of pertechnetate would be sensed by SPR.
Poly(ethylene glycol), PEG, derivatized gold surfaces were prepared via modification of a published procedure for the formation of selfassembled monolayers (SAMs) from short chain oligo(ethylene oxide) based thiols $\mathrm{HS}\left(\mathrm{CH}_{2}\right)_{11}\left(\mathrm{OCH}_{2} \mathrm{CH}_{2}\right)_{\mathbf{n}} \mathrm{OR}(\mathrm{n}=1-$ 17; $\mathrm{R}=\mathrm{H}, \mathrm{Me} ; \mathrm{MW}<1000)^{30}$. These short chain thiols are reported to be soluble in ethanol and stable in air. The longer chain PEG-thiol used in this study was not soluble in anhydrous ethanol. Consequently, a PEG-thiol mixed

solvent system of anhydrous ethanol with methylene chloride added for increased solubility was used in initial studies. It was not clear from the literature as to why or even if it was necessary for the ethanol to be anhydrous for the formation of oligomeric PEG-thiol based SAMs. Water was reported to be a suitable solvent for the functionalization of gold surfaces with orthopyridyl-disulfide terminated PEGs, OP-SSPEG, (MW = 2000-5000) and was tested as a solvent in these studies with the higher 
molecular weight PEG as well. Also, the longer chain PEG-thiol used in our studies reacts with oxygen to form a disulfide (PEG-SS-PEG). ${ }^{2}$ Because it was not clear if such a sterically bulky disulfide would be expected to form a monolayer similar to the OP-SSPEG, additional precautions were taken for the exclusion of oxygen from the PEG-thiol reaction solutions to avoid formation of additional disulfide, PEG-SS-PEG, (some disulfide is present as an impurity in the material as received from the vendor).

A significant degree of variability was found in the literature for the preparation of the gold surface. Both sputtering and evaporation techniques for gold deposition are reported. Furthermore, a variety of gold surface cleaning procedures were reported ranging from no cleaning if the gold substrate was used immediately after preparation to mild cleaning by washing with organic solvents to severe cleaning requiring peroxide/nitric acid solutions. We chose mild cleaning with ethanol and deionized water for substrates exposed to PEG solutions.

The formation of PEG monolayers on gold coated silicon wafers was investigated using spectroscopic ellipsometry. Initial optical constants were determined for the gold layer on substrate \#1 and were found to be consistent with the gold optical parameters used as reference values in the ellipsometric software. The film appears to be optically thick as the use of a silicon wafer substrate in the modeling does not change the fit of experimental to model generated data. The standard gold reference value obtained from the Wvase32 software package is $n=0.166$ and $k=3.178$ at $654 \mathrm{~nm}^{31}$. The optical constants determined by fitting the psi and delta ellipsometric parameters from $600-100$ $\mathrm{nm}$ were measured to be $n=0.154$ and $k=3.78$ at $654 \mathrm{~nm}$. This difference is most likely due to the gold film deposition parameters, where a sputtered film exhibits slightly different optical constants than an evaporated film. However, the constants match closely enough for our purposes where the substrate and gold film optical properties were determined and then used in subsequent analyses.

The Si/gold substrate \#1 was removed from the PEG-SH ethanol/dichloromethane solution and immediately rinsed in methylene chloride, ethanol and deionized water. After drying in a nitrogen jet, the substrate was mounted and aligned and the ellipsometric parameters collected using 50 revolutions $/ \mathrm{s}, 30^{\circ}$ polarizer angle, and high resolution mode. The scatter in the ellipsometric thickness is approximately $2 \AA$ for repeated mounting and analysis of the $\mathrm{SiO}_{2}$ calibration sample. Subsequent mounting of prepared silicon wafers is expected to have this type of scatter, provided the thin films are of uniform thickness around the center of the substrate. After each measurement, the substrate \#1 was removed, washed in ether deionized water or ethanol, and then remounted and analyzed. Data were collected and analyzed using the Wvase 32 analysis software and a variety of models in order to obtain the best fit with the most physically representative model. Initial fitting was performed with a simple Cauchy layer, where thickness and real refractive index (Cauchy parameters) were allowed to vary. It was found that this yielded non-physical results with a negative second coefficient. Other PEG ellipsometric studies found in the literature use a bulk refractive index of 1.45 and simply fit adlayer thickness using a three layer model ${ }^{32}$ consisting of gold, PEG, and air. Therefore, the Cauchy coefficients were fixed at 1.45 and .01 , and the thickness fit for each subsequent washing. It was found that the values of the refractive index do not 
significantly affect the thickness prediction, and this is consistent with the fact that ellipsometry is not as sensitive to material optical constants for very thin films $(<50$ Angstroms). However, the fit was improved somewhat by the inclusion of a small absorption component to the model. The fit results are given in Table 2. The MSE value is a measure of the fit of experimental to model data, and in general lower MSE's are better. An initial thickness of $56 \AA$ is consistent with a multiple monolayer coverage of PEG 5000, with a chain length of 114 monomer units yielding a molecular chain length of approximately $32.6 \AA$ if the molecule is extended completely from the surface ${ }^{29}$. Successive washing of the substrate yielded a decrease in calculated film thickness and refractive index (see table 2). The index and thickness correlation is most likely due to removal of material but could be due to some extent from reorganization of the PEG on the surface due to solvent effects during washing and drying. If PEG is being removed from the surface, it is not clear if this is merely unreacted PEG-thiol adhered to the surface that is eventually washed away or rather the bound PEG thiolate on the gold surface is removed as the thiol in a reversible process or possibly as a PEG-disulfide dimer (PEG-SS-PEG) resulting from coupling of two thiolate-PEGs. Deviations in 1:1 compositions for SAMs formed from asymmetric alkyl disulfides have been reported, which suggest that exchange processes at the surface are likely. In this case, the lower surface coverage would yield a less dense optical layer, and this would yield lower refractive index values for the layer.

The imaginary refractive index, $k$, which is a measure of the optical absorption of the film, increases with decreasing real refractive index, and it is unclear if this is a result of real absorption increase or is simply an artifact of the real refractive index change. The fact that the thickness change is insensitive to changes in the refractive index indicates that the change in thickness, or apparent thickness, is real and due to removal of material from the surface. This interpretation of the refractive index data is difficult to justify from an ellipsometric point of view, as ellipsometric parameters are relatively insensitive to the refractive indices of very thin layers. However, the trend to lower index values generally supports the idea that the surface coverage is decreasing with each wash. The refractive index for analysis \# 8, measured the following day after exposure to ambient air overnight without a wash prior to mounting, was greater than the initial refractive index of the film, while the film thickness was essentially unchanged. This is an indication of film rearrangement or densification in air. Literature references cited in this section do not discuss film longevity as the reported adlayer characterizations were performed immediately after processing following a single rinse to remove unbound PEG.

An additional PEG adlayer was formed on gold coated silicon wafers by placing the disk in a polyethylene bottle with $1 \mathrm{mmol} / \mathrm{L}$ PEG-SH dissolved in previously degassed deionized water for 7 days. The disk was removed and adlayer optical properties measured by ellipsometry as above. The layer thickness was reduced from an initial 18.5 $\AA$ to $9.9 \AA$ after 4 washings. This again illustrates that the PEG layer was formed and then was removed by subsequent washing. The difference in starting layer thickness could be attributed to difference in solvent (EtOH/dichloromethane vs. water). However, the final coverage of PEG on both substrates after washing was similar to reported coverages of 5000 molecular weight methoxy-PEG orthopyridyl-disulfide [ref. 
29]. The PEG surface coverage will be discussed additionally in the SPR sensor characterization section as other investigators have researched PEG thiolate linkages to gold using SPR.

Table 2. Refractive index and thickness measurement results for Sigold \#1 wafer, successive washes after formation of $P E G$ adlayer.

\begin{tabular}{|c|c|c|c|c|}
\hline \# of washes & Thickness, $\AA$ & $n @ 654 \mathrm{~nm}$ & $k$ amplitude & Thickness, index held constant \\
\hline 1 water & $56.64 \pm 0.91$ & $1.4485 \pm 0.0006$ & $0.157 \pm 0.010$ & $56.65 \pm 0.05, \mathrm{MSE}=2$ \\
\hline 2 water & $32.77 \pm 0.05$ & $1.4420 \pm 0.001$ & $0.231 \pm 0.002$ & $32.775 \pm 0.26, \mathrm{MSE}=10.51$ \\
\hline 3 water & $29.60 \pm 0.12$ & $1.4379 \pm 0.002$ & $0.261 \pm 0.004$ & $29.58 \pm 0.34, \mathrm{MSE}=11.12$ \\
\hline 4 water & $28.31 \pm 0.10$ & $1.4346 \pm 0.002$ & $0.282 \pm 0.004$ & $28.265 \pm 0.39, \mathrm{MSE}=13.83$ \\
\hline 5 EtOH, water & $25.01 \pm 0.10$ & $1.4306 \pm 0.002$ & $0.307 \pm 0.005$ & $24.916 \pm 0.40, \mathrm{MSE}=17$ \\
\hline 6 EtOH, water & $18.29 \pm 0.22$ & $1.4126 \pm 0.004$ & $0.396 \pm 0.009$ & $18.278 \pm 0.51, \mathrm{MSE}=15.74$ \\
\hline 7 EtOH, water & $16.62 \pm 0.09$ & $1.4072 \pm 0.004$ & $0.416 \pm 0.008$ & $16.431 \pm 0.50, \mathrm{MSE}=14.47$ \\
\hline 8, no wash* & $15.92 \pm 0.35$ & $1.4454 \pm 0.003$ & $0.227 \pm 0.007$ & $15.974 \pm 0.13, \mathrm{MSE}=6.04$ \\
\hline 9 EtOH, water* & $11.04 \pm 0.16$ & $1.4287 \pm 0.004$ & $0.354 \pm 0.010$ & $11.048 \pm 0.23, \mathrm{MSE}=9.64$ \\
\hline
\end{tabular}

* analyzed the next day after sitting in ambient air overnight

\section{Surface Plasmon Resonance Sensor Performance}

\section{SPR Probe Optical Performance: Integrity of the SPR Spectrum}

An SPR experiment requires that the optical reflectivity be measured at the sensor/liquid interface, and this in turn requires some reference beam which approximates the lamp intensity after it has traversed the optical system. This can be achieved with the SPR probe by exposing the sensor to air, which does not generate a plasmon resonance, and recording the reference spectrum prior to performing liquid experiments. The probe design is such that rotation of the hemisphere, which is held in place by a screw cap sealed with Viton o-rings, causes slight shifts in the resonance due to imperfections in the machining of the probe as well as non-uniformity in the gold layer. It is important, therefore, that the screw-cap be tightened sufficiently and that the referencing procedure be performed in the cell, after the Swage-Loc fittings have been tightened. This procedure provides a reference spectrum which is representative of the optical system and therefore increases the quality of the SPR spectrum obtained when sample is passed over the sensor.

Figure 4 displays a comparison between the SPR spectrum obtained in deionized water at $30^{\circ} \mathrm{C}$ with a theoretical spectrum calculated from the material optical constants, gold thickness, and refractive index of water. The theoretical spectrum was calculated from known optical constants for the materials making up the three layer optical structure (alumina, gold, and water) using a Matlab ${ }^{\mathrm{TM}}$ script based on the calculation of the Fresnel reflection coefficients for the structure using a matrix formalism ${ }^{33}$. The gold thickness was varied during modeling in order to determine by comparison the actual gold 
thickness on the hemisphere. The SPR sensor gold thickness determined from the position of the resonance is approximately $440 \AA$ while the target deposition thickness was $550 \AA$. The $11 \mathrm{~nm}$ difference between the measured and target thicknesses can be attributed to errors in the thickness monitor due to proximity of the sample and thickness monitor inside the vacuum chamber during deposition.

Experimental SPR transmission spectrum referenced to air, mixed polarization

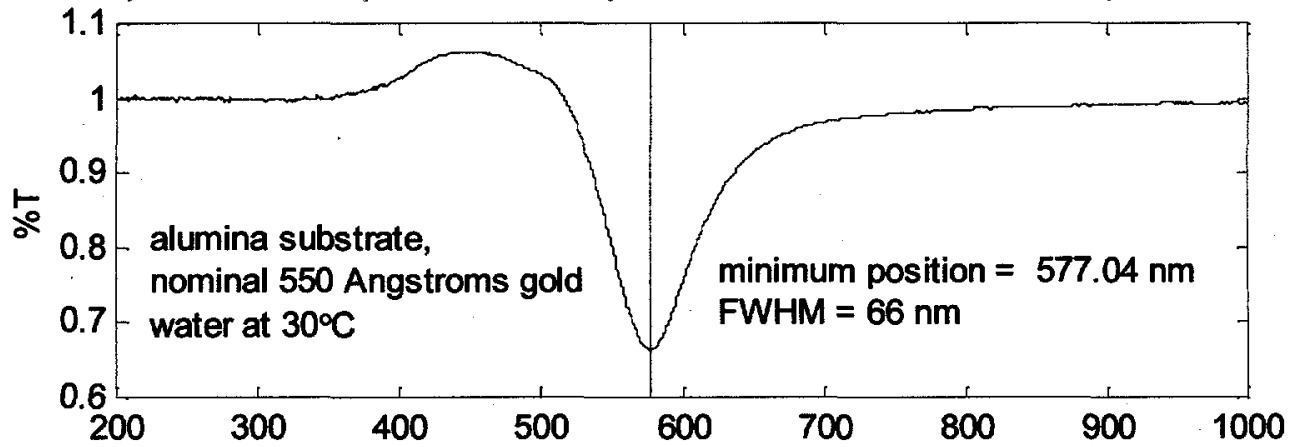

Theoretical SPR transmission spectrum referenced to air, p-polarization

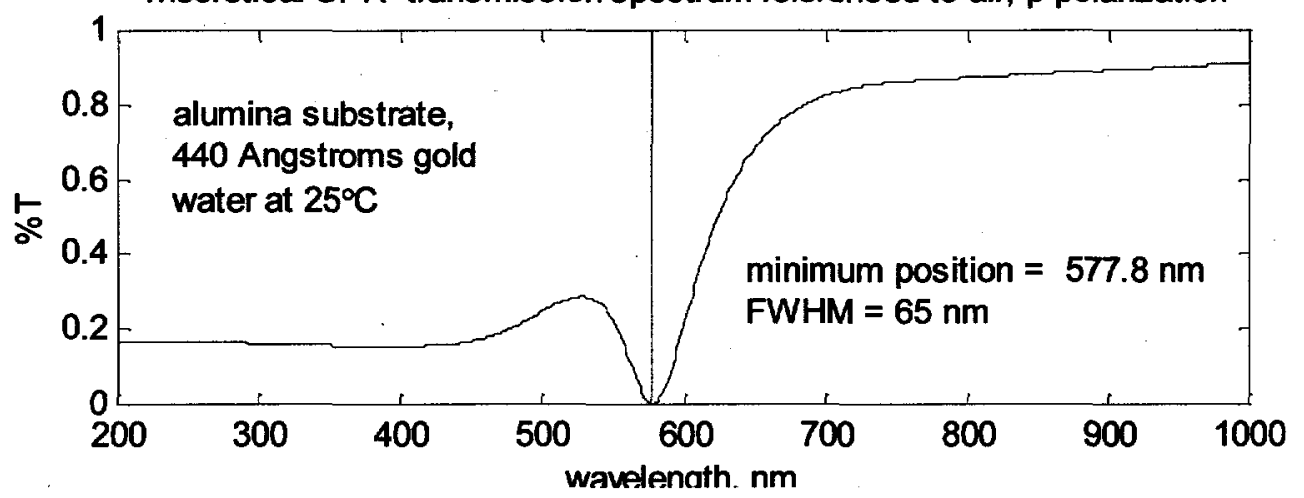

Figure 4. Comparison between experimental SPR spectrum (above) and theoretical spectrum (below) shows that the SRTC fiber optic SPR probe generates high quality, high resolution spectra.

The difference in magnitude of the resonance between the experimental and theoretical plots is due to the nature of the optical system and the referencing method. The calculation to obtain the theoretical curve is analogous to a reflectivity calculation, or simply $\mathrm{I} / \mathrm{I}_{0}$, where the source intensity profile is taken as $\mathrm{I}_{0}$. Therefore, the reflectivity is a convolution of the SPR feature, shown as a sharp minimum at around $580 \mathrm{~nm}$, and the reflectivity of gold metal, which is lower in the blue end of the spectrum due the absorption of gold, which gives it its yellow hue. The experimental curve was generated with a real reference of the optical system through the SPR probe and the reference spectrum, therefore, was attenuated by the gold at lower wavelengths. This is why the SPR spectrum from the SPR probe referenced to air is symmetrical and the reflectivity approaches unity away from the resonance. In addition, the establishment of a resonance requires that the polarization state of the input beam contain transverse magnetic components (TM or p-polarized). If the polarization state is pure, and the metal film is the correct thickness, the reflectivity at the resonance approaches zero. The theoretical 
calculations used in the generation of the theoretical curve assumes pure p-polarized light. Conversely, the experimental SPR spectrum was obtained using a white light source with no polarizing optics. The orthogonal polarization states, transverse electric (TE, or s-polarized) and transverse magnetic, p-polarized, are present in roughly equal proportion due to the isotropic and random nature of the tungsten emission. In addition, any stable polarization state emitted from the lamp would be lost in the fibers due to randomization in the fiber core. The p-polarized components of the incident light would excite the plasmon oscillation while the s-polarized light return unmodified to the detector. Therefore, the reflectivity minimum limit for 50:50 mixed polarization is 0.5 . The minimum reflectivity in the experimental spectrum is approximately 0.65 , indicating that some polarization of the beam is occurring, most likely due to the small air gap between the fiber end face and the hemisphere flat.

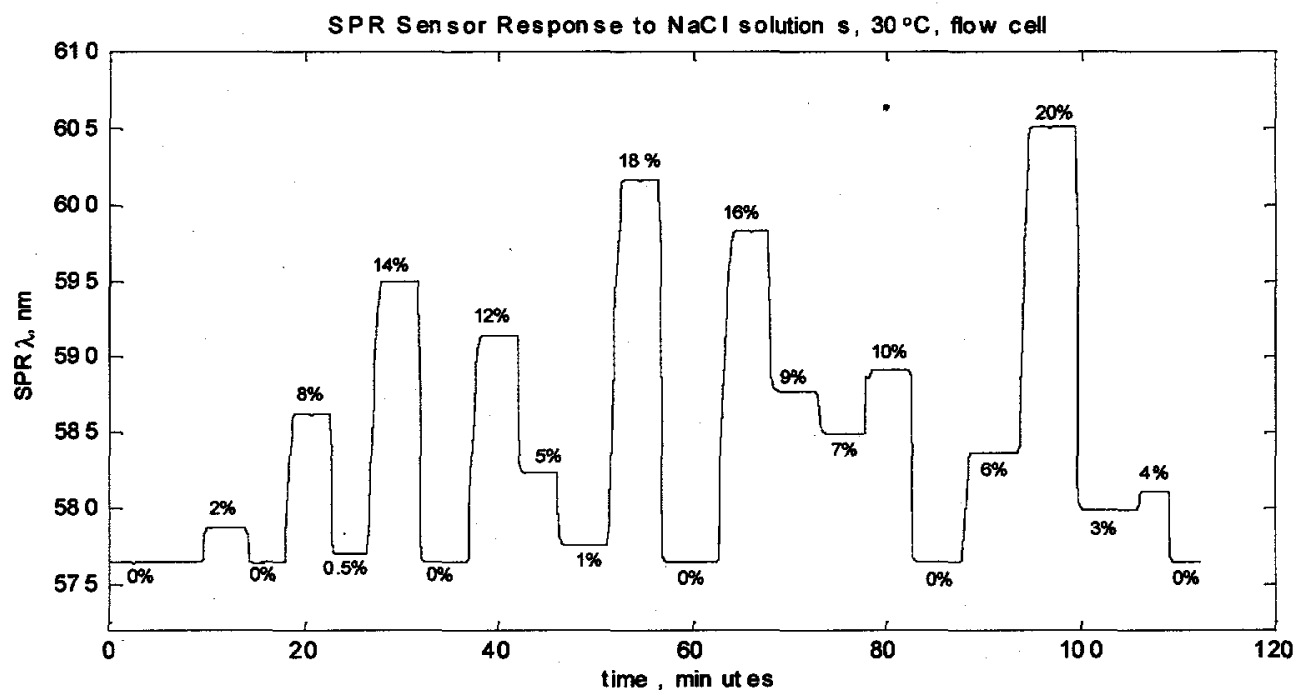

Figure 5. SPR sensor response to a series of sodium chloride solutions shows the low noise, high sensitivity response of he SRTC SPR Probe. The solutions were run in random order with the pump stopped intermittently to test for cell holdup.

A key measure in determining the quality of the SPR spectrum is the full width at half maximum (or minimum) (FWHM) of the resonance. Broadening of the resonance is generally caused by poor collimation of the incident beam in white light experiments. The curvature of the hemisphere compensates for the divergence of the optical fibers and fixes the incident angle at $60^{\circ}$, with very little off-axis light striking the interface. The fact that there is essentially no broadening in the resonance with respect to the theoretical FWHM indicates the probe is generating high resolution, low noise SPR spectra. This is important in that the limit of detection for surface adsorbed species is a function of how well one determines the position of the resonance. The ability to distinguish small shifts in the resonance degrades as the resonance broadens.

\section{Flow Cell Bulk Refractive Index Calibration}

A major characterization step for any new SPR technique is to evaluate the bulk refractive index response of the sensor to a simple chemical system in order to determine 
sensitivity and stability. Sodium chloride was used as the refractive index perturber as literature values for weight percent composition in water were readily available. The refractive indices for the solutions were calculated, rather than measured, due to a lack of a reference method (Abbé refractometer). We believe that the refractive index values are accurate based on the accuracy of the gravimetric method used to prepare them. The SPR probe was introduced into both static and flowing systems for bulk refractive index characterization. However, the flow system results are presented here as all SPR PEG monolayer formation experiments were performed under temperature controlled flow.

Figure 5 shows the real time flow response of the sensor to the $0-20 \%$ wt. $/ w t$. sodium chloride salt solutions where data were acquired in 10 second intervals. The solutions were analyzed randomly with the pump stopped for each solution change and then stopped again during each sample exposure in order to test for cell holdup. The solutions very quickly reached the flow cell (within a minute) and the step change in SPR response exhibited for each sample indicates the cell holdup was minimal. Stable SPR positions were used in a second order polynomial lest squares fit of the refractive index at $30^{\circ} \mathrm{C}$ to the SPR wavelength values for each solution. Figure 6 displays the relationship between the SPR wavelengths and the refractive indices of the various calibration solutions. The curve is consistent with the non-linear response characteristic of SPR sensors ${ }^{35}$. As the resonance wavelength increases, the sensitivity increases as well. The probe sensitivity to refractive index may be calculated from the regression coefficients around a specific wavelength, which for small shifts is essentially linear. The sensitivity calculated near the refractive index of water is $9.9 \times 10^{-4}$ refractive index units (RIU) per $\mathrm{nm}$, or $1010 \mathrm{~nm} / \mathrm{RIU}$. This slope increases with SPR wavelength, and operation at longer wavelengths would provide increased sensitivity to changes in refractive index. However, this sensitivity is comparable to other SPR systems reported

SPR temperature controlled calibration with $\mathrm{NaCl}$ solutions

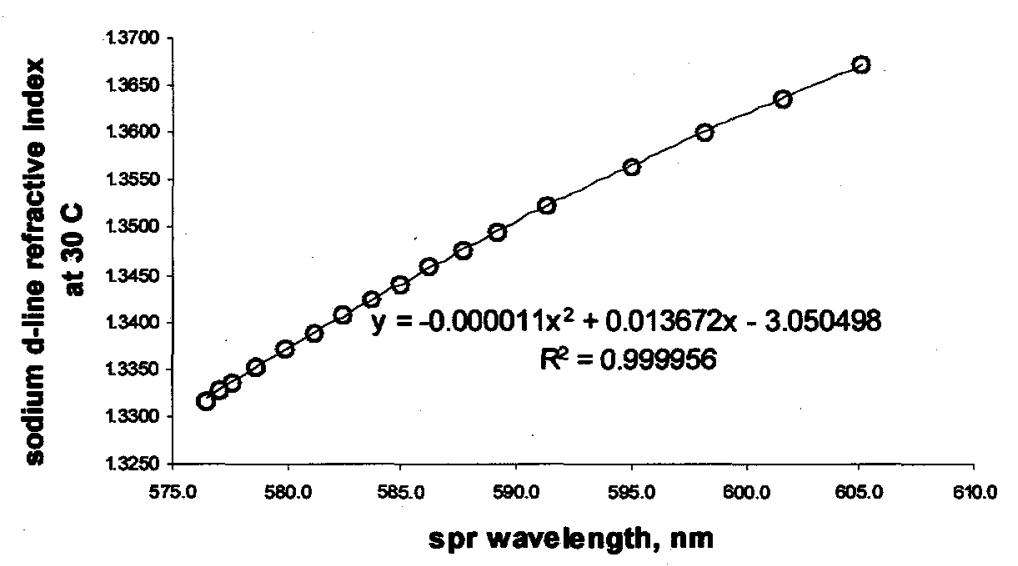

Figure 6. SPR sensor refractive index calibration results show the non-linear relationship between the SPR wavelength and refractive index of the sample. The probe has a sensitivity of $1010 \mathrm{~nm} / \mathrm{RIU}$ for a refractive index range close to that of water. in the literature ${ }^{4}$ and is suitable for surface absorption studies.

The optical system stability was evaluated in order to determine the system sensitivity to changes in the resonance wavelength. Figure 7 shows the SPR wavelength position measured during a single 30 minute period under temperature control with water flowing through the system at $1.6 \mathrm{ml} / \mathrm{min}$. The standard deviation of the SPR position, which is a measure of the noise in the system, is $0.001 \mathrm{~nm}$, and this is due to the stability of the optical 
spectrometer, light source, and peak fitting algorithm used to find the resonance position. This short term stability, coupled with the calibrated refractive index sensitivity reported above, indicates that the SPR probe can differentiate changes in bulk refractive index of $9.9 \times 10^{-7} \mathrm{RIU}$. This is comparable to reported sensitivity values for high resolution, benchtop SPR apparatus and is one to two orders of magnitude more sensitive than the fiber optic dip probe commercially available from Pharmacia, Inc. When compared with sensor systems reported in the literature, the sensitivity and stability of the SRTC SPR probe are sufficient to monitor the formation of the PEG adlayer and sensor changes in the adlayer which are due to surface binding of analyte, in this case the non-radioactive pertechnetate surrogate, perrhenate.

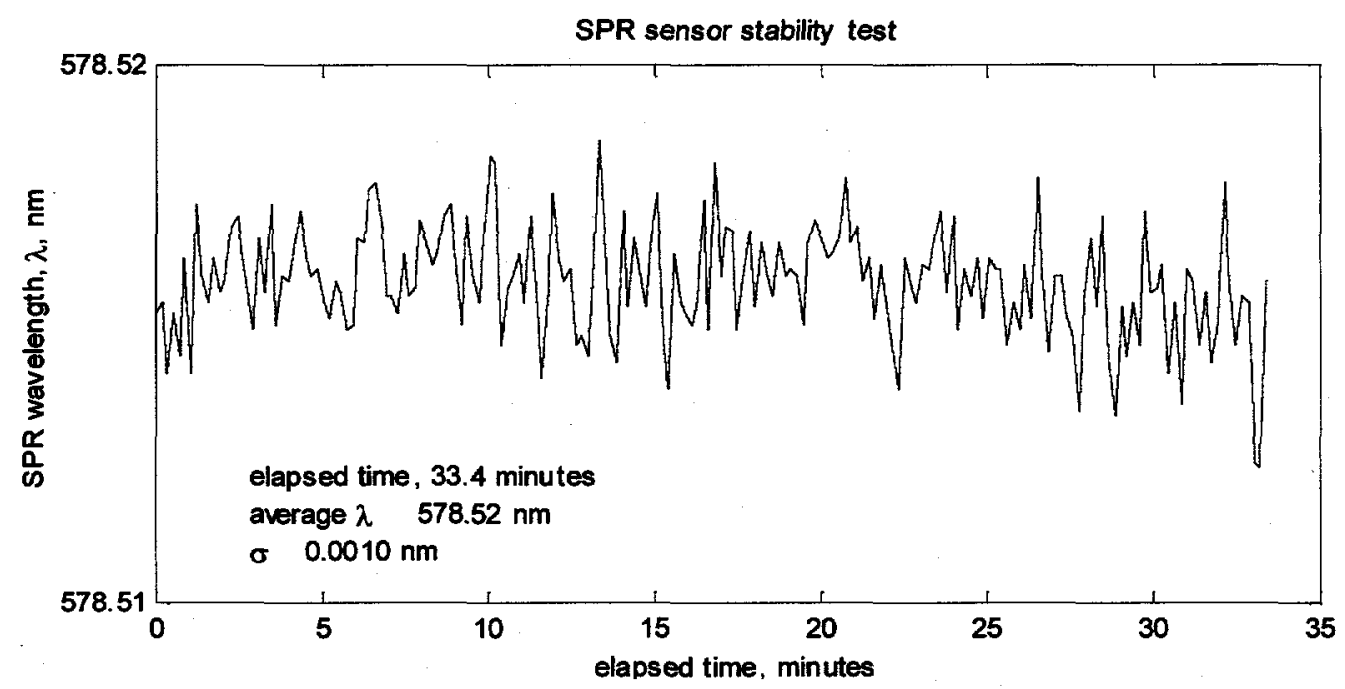

Figure 7. SPR sensor temperature stabilized response to circulated deionized water shows excellent stability for short term experiments and provides a baseline for determining the minimum detectable change in bulk refractive index.

The characteristics of the flow system and cell affect the stability of the SPR experiment. During the salt calibration run, and all subsequent experiments, the probe was referenced with air in the cell and then allowed to equilibrate with the liquid flow. For gold surfaces which had been exposed to ambient air conditions for greater than a few hours prior to exposure to liquid, the SPR wavelength at liquid flow initiation was not stable but rather decreased exponentially to a baseline value after approximately 4 hours. The magnitude of the decline was approximately 0.5 to $0.7 \mathrm{~nm}$, and was evident both in static, dip environments as well as in the flow cell system. The origin of the initial decrease in SPR wavelength is unknown, but it may be due to removal of surface contaminants or hydration of the probe. At times the system was re-referenced between experiments by purging the flow cell with air and then reintroducing deionized water into the cell. These steps did not result in the same initial decrease in SPR wavelength. Therefore, it appears that the system relaxation occurred as a result of long term exposure to ambient air, which lends credence to the theory that removal of surface contaminants during initial exposure to liquid is the cause of the initial SPR response. 
Ambient temperature in the laboratory was between $22^{\circ} \mathrm{C}$ and $25^{\circ} \mathrm{C}$ while the incubator control was set at $30^{\circ} \mathrm{C}$ for flow experiments. The pump was set above the incubator and solution was pumped out of and back into the incubator. At a constant pump rate, the fluctuations in the signal are low, as in figure 7 , and this indicates that the system is in temperature equilibrium during flow, with the solution in contact with the SPR sensor at a slightly lower temperature than the cell. In addition, the incubator temperature fluctuated with laboratory temperature and with the opening and closing of the chamber door during sample change-out. Figure 8 shows the SPR system response to pump cycling.

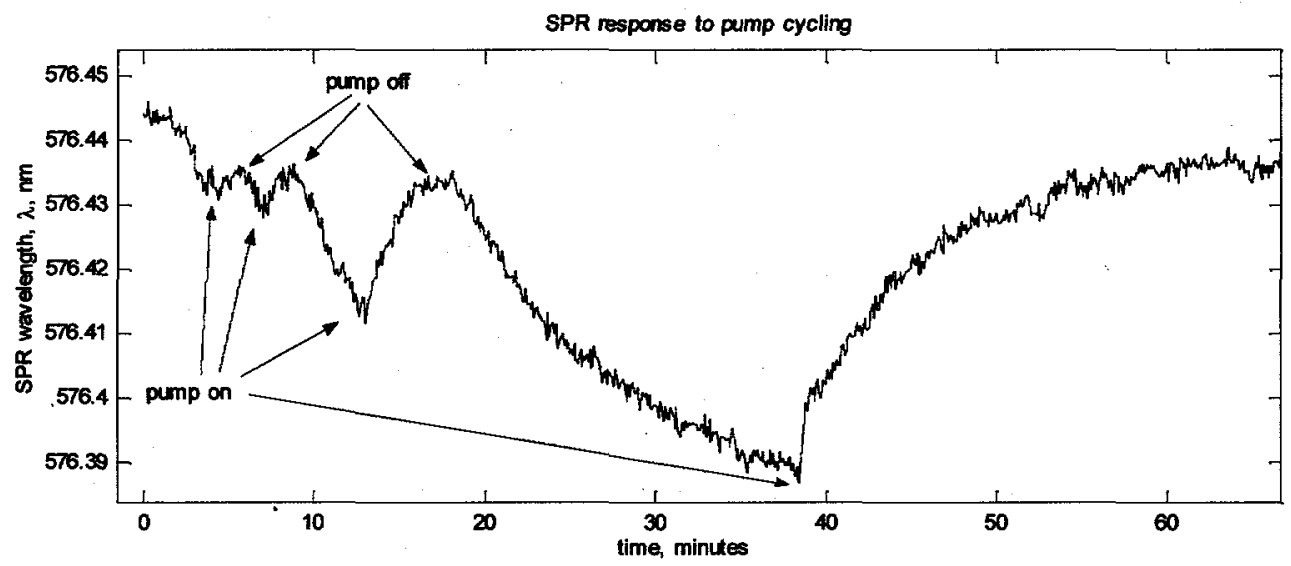

Figure 8. SPR sensor response to sequential flow stoppage. The negative trend upon flow stoppage is due to temperature equilibration within the flow cell, where the calculated temperature difference between the liquid flow and the SPR sensor and flow cell is approximately $0.43^{\circ} \mathrm{C}$.

For each time the pump was shut off, the SPR position decreased, indicative of an increase in solution temperature as the temperature dependence on the refractive index of water is $\frac{\partial n}{\partial T}=-1.17 \times 10^{-4} /{ }^{\circ} \mathrm{C}^{34}$. The negative dispersion of water with temperature would yield a decrease in SPR wavelength as the liquid attained thermal equilibrium at the higher incubator temperature. The small SPR shift (on the order of $0.05 \mathrm{~nm}$ ) during the long pump stoppage corresponds to a temperature change of $0.43^{\circ} \mathrm{C}$, which indicates that the sample is not entirely temperature equilibrated as it reaches the flow cell.

Lengthening the tubing inside the incubator or increasing the residence time in the tubing by slowing the pump speed would, in theory, mitigate the temperature differences between the pump and the cell. This is why the experiments were performed in constant flow, with pump stoppage only for the few seconds required to change sample bottles. Long term data acquisition was affected slightly by the laboratory temperature, as will be demonstrated in the next section.

\section{In-Situ Poly(ethylene g/ycol) Film Formation}

Two separate temperature controlled PEG experiments were performed with the aim of assembling, in-situ, the monolayers of PEG on the gold surface of the SPR probe. The 
first was performed using the $0.1 \mathrm{mmol} / \mathrm{L}$ PEG-SH solution. The PEG was introduced into the flow system and then re-circulated overnight. Figure 9 shows the SPR response to circulation of the PEG solution with the temperature fluctuation plotted below for comparison. The small slope change in the SPR response at around 9 hours elapsed time is due to the temperature fluctuations in the lab, as the SPR response is anticorrelated with temperature. The first hour of data acquisition (temperature and SPR response) is plotted in Figure 10 and shows the initial large shift $(0.1 \mathrm{~nm})$ due to rapid binding of PEG. The temperature response clearly shows the opening of the incubator door followed by the re-equilibration of the temperature inside the block. The SPR response also contains a slight temperature component, and one would think that the SPR response is due solely to temperature. However, the temperature drops associated with opening the door at $t=6$ minutes and $t=19.5$ hours are similar in magnitude while the SPR responses are different, $(0.1 \mathrm{~nm}$ shift at $\mathrm{t}=6$ minutes, $0.01 \mathrm{~nm}$ at $\mathrm{t}=19.5$ hours $)$ indicating that the response at $t=6$ minutes is primarily due to the PEG binding on the SPR gold surface. Following the initial response to rapid binding of PEG, the SPR sensor showed response over a 24 hour period. The sensor response asymptotically approached a resonance shift of $0.5 \mathrm{~nm}$, and this is consistent with what other researchers have observed with PEG films formed by thiolate linkages to gold: a short term, rapid response followed by a longer term assembly of the remainder of the monolayer or additional monolayers ${ }^{36}$.

The surface layer thickness and degree of coverage may be estimated by applying a model which relates the known SPR parameters with parameters which describe the adlayer by the following equation ${ }^{35}$

$$
d=-\left(\frac{\ell_{d}}{2}\right) \ln \left(1-\frac{R}{R_{\max }}\right)
$$

where $d$ is the estimation of the adsorbed layer thickness for a uniform monolayer, $\ell_{d}$ is the evanescent field wave decay length determined by the sample optical properties of the SPR sensor and sample, $R$ is the measured SPR response, and $R_{\max }$ is the maximum response (SPR shift in $\mathrm{nm}$ ) measured for an infinitely thick layer. $\ell_{d}$ may be roughly estimated at $37 \%$ of the SPR probe wavelength ${ }^{36}$, which is $213 \mathrm{~nm}$ for a probe wavelength of $575 \mathrm{~nm}$. Once the monolayer thickness has been calculated, the surface coverage may be estimated from

$$
\theta=d \times N
$$

where $\theta$ is the number of molecules per $\mathrm{cm}^{2}$ and $\mathrm{N}$ is the number density of molecules estimated from the bulk density of the material, which for PEG is $1.125 \mathrm{~g} / \mathrm{cm}^{3}$.

The maximum response, or change in measured SPR wavelength, is calculated from the difference in refractive index between water and the adlayer material. Using 1.3317 and 1.4087 for the refractive indices of water and PEG, respectively, and applying the calibration results obtained from the salt solutions, yields $R_{\max }$ of $77 \mathrm{~nm}$. Therefore, the surface layer thickness estimated for the initial adsorption phase is approximately 0.138 
BNF-003-98-0308

June 30, 2000

Page 21 of 30

$\mathrm{nm}$, or $1.38 \AA$. This corresponds to a surface coverage, $\theta$, by equation 2 , of $1.49 \times 10^{12}$ molecules $/ \mathrm{cm}^{2}$ which yields an initial surface density of $15.52 \mathrm{ng} / \mathrm{cm}^{2}$, which is an order of magnitude lower surface density than that reported by $\mathrm{Lu}$, et $\mathrm{al}^{36}$ for a PEG -5000 linked to a gold surface via a thiolate bond. This indicates that the initial surface coverage is poor, with significant space between attached PEG molecules. In addition, the investigators have shown that the surface coverage measured by SPR in flow cell experiments is considerably lower than the expected coverage for PEG molecules of significant molecular weight. In their studies, the larger molecular weight PEGs ( $\sim 5000$ M.W.) had the lowest surface coverages, and this is attributable to steric hindrance to additional attachment once the initial, rapid attachment had occurred, and our results are consistent with their interpretation.
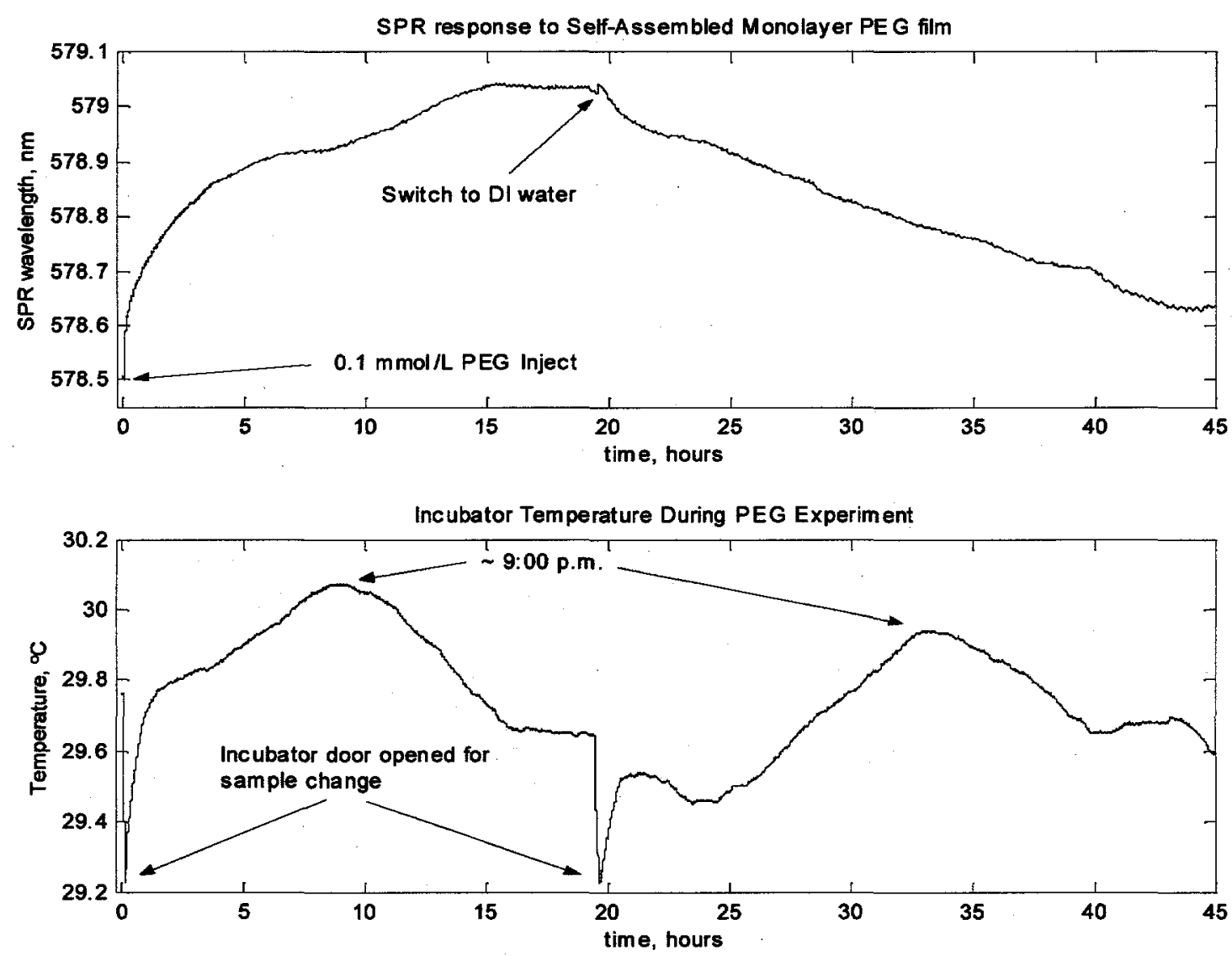

Figure 9. SPR sensor response to adsorption of monomethylated PEG-thiol (M.W. $=5000)$ (above) and temperature fluctuation during the experiment (below).

The longer term phase of attachment, occurring over the course of 24 hours, is consistent with $\mathrm{Lu}$ 's results as well, where the longer term assembly was verified by Xray photoelectron spectroscopy to have roughly doubled the surface coverage. At 19.5 hours the SPR response leveled out indicating that the monolayer assembly was complete. The final monolayer thickness and surface coverage are $6.9 \AA$ and 77.6 $\mathrm{ng} / \mathrm{cm}^{2}$, and this is again consistent with the interpretation that a second, long term phase of assembly results in thicker coverage and is also comparable with the ellipsometric 
results after the final washing. However, the assembly was monitored by SPR over 24 hours. In general, the other researchers cited in this report have monitored the initial phase of assembly by SPR and then used ex-situ methods to determine the final thickness after 1 to 6 days of assembly following a rinse step to remove weakly bound PEG. The work reported here appears to be the first in-situ SPR study of the extended phase of assembly of PEG adlayer on gold, which was made possible by the low noise SPR sensor system developed by SRTC.
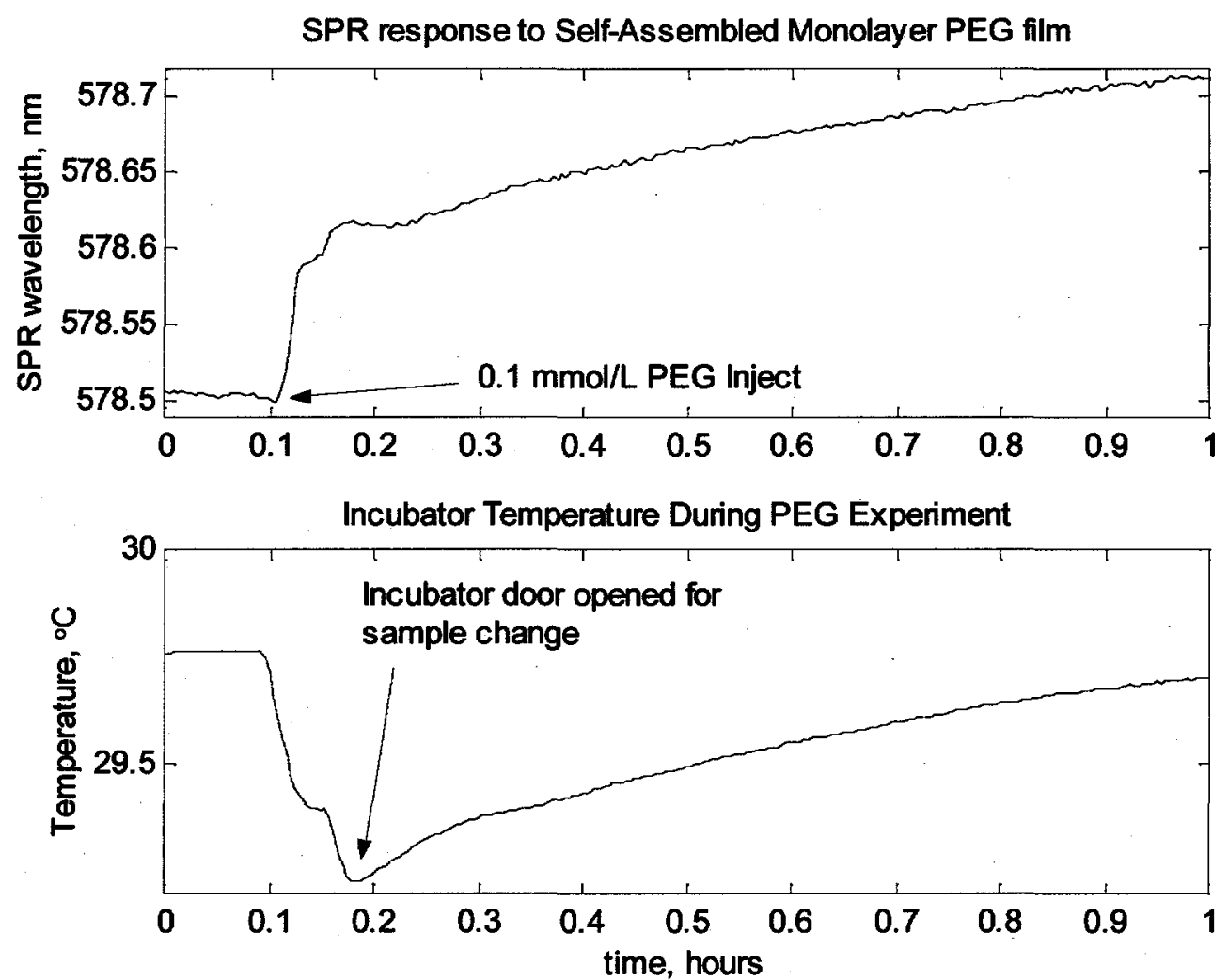

Figure 10. First hour of PEG adlayer formation (above), as shown in figure 9, where the temperature fluctuation is shown (below).

A significant finding from this extended flow experiment is the slow, monotonic decrease in SPR resonance wavelength after the flow system was switched to deionized water that we have monitored by both SPR and spectroscopic ellipsometry. As mentioned earlier, literature reports do not discuss the long term stability of PEG films. As can be seen in figure 9, the PEG layer did not immediately detach. This indicates that the film is either reorganizing or suffering from removal of material. The PEG layer thickness decrease could be due to shear forces in the flow or to the possibility that the second phase of PEG assembly is a physisorption process, rather than a chemisorption process. The final SPR wavelength position supports this interpretation, as the initial 0.1 $\mathrm{nm}$ shift is retained, indicating that the PEG attached to the gold surface in the initial, rapid, assembly phase is chemisorbed. It is unclear from these data what the true mechanism behind the disassembly is, but we have demonstrated it in both the ellipsometric and SPR data. Further study is required regarding the robustness of large 
chain PEG films attached via thiolate linkages to gold surfaces. However, it appears that the PEG film does remain, to some extent, and this may be suitable for SPR sensing of pertechnetate, or a non-radioactive chemical surrogate, if the PEG film which remains is sufficiently dense enough to support aqueous biphasic behavior.

\section{PEG Functionalized SPR Sensor Response to Perrhenate}

The second PEG attachment experiment was performed as the first using freshly prepared $0.11 \mathrm{mmol} / \mathrm{L}$ PEG in deionized water. The PEG solution was circulated through the flow cell once the SPR sensor had stabilized (this sensor was immediately placed into the flow cell after vacuum coating). The PEG adlayer was assembled for 24 hours and resulted in a similar adlayer thickness and surface coverage as achieved with the initial PEG SPR experiment. The system was switched over to the $4 \mathrm{M} \mathrm{NaOH}$

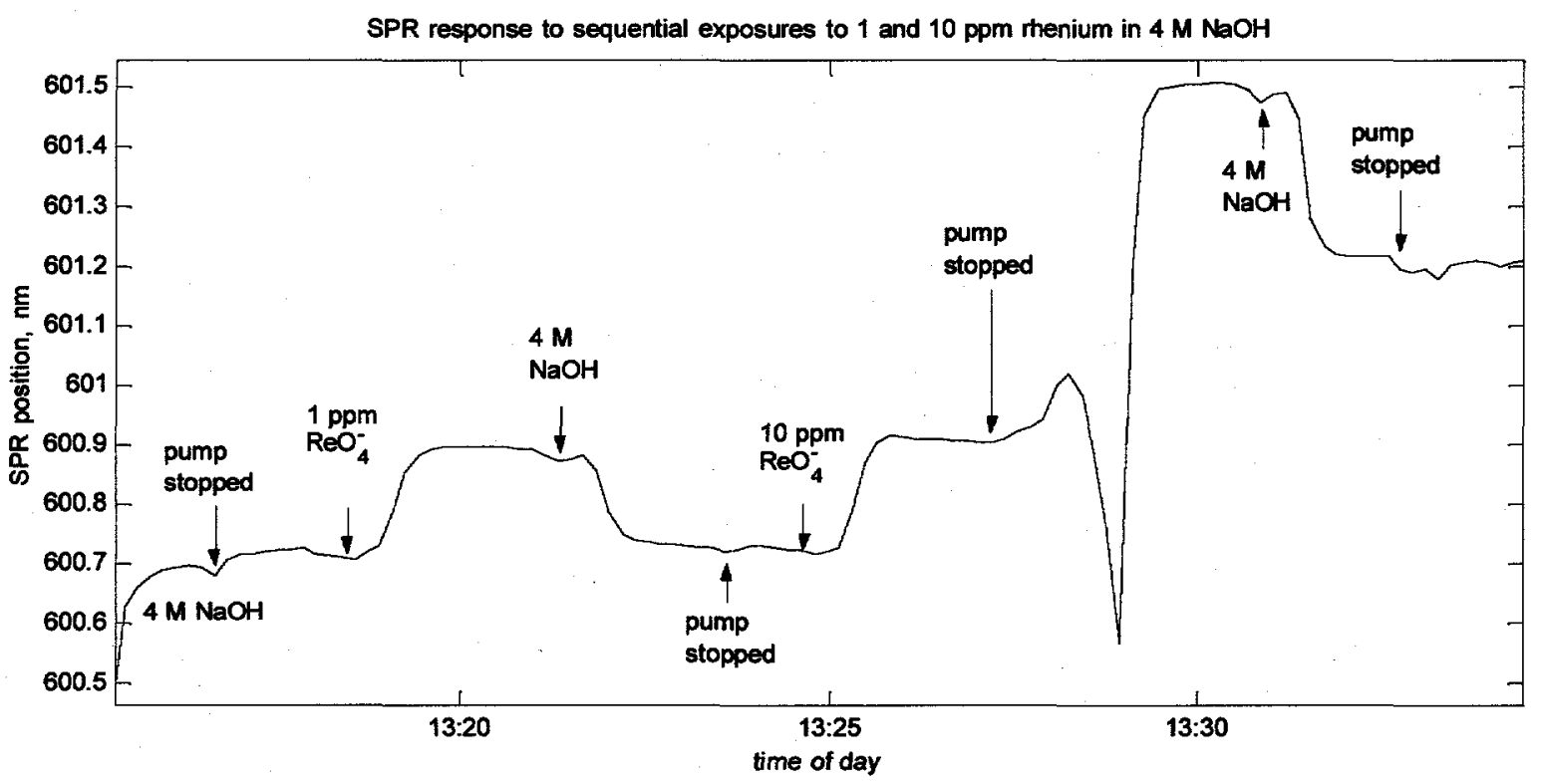

Figure 11. SPR sensor response to4 $M$ NaOH blank and $4 M \mathrm{NaOH}$ with 1 and 10 ppm perrhenate. The initial exposures are shown here so the detail in the perrhenate response can be seen.

simulant and immediately responded with a large SPR shift ( $>35 \mathrm{~nm})$. However, a bubble had been inadvertently introduced or generated in the tubing and required referencing of the system in air and then introduction of deionized water followed again by the $\mathrm{NaOH}$ blank. After stabilization, the $\mathrm{NaOH}$ blank was again introduced into the flow cell and the resonance shifted from $573 \mathrm{~nm}$ to $600.7 \mathrm{~nm}$. The smaller response to $\mathrm{NaOH}$ after referencing is unexplained, as the solution composition would not have changed significantly. It is possible that temperature effects in the cell or some other surface effect caused the initial large shift and subsequent bubble formation.

Nevertheless, after the system was re-referenced in air and stabilized in deionized water and then $\mathrm{NaOH}$, the SPR sensor was exposed to perrhenate solutions in order to test the response. 
BNF-003-98-0308

June 30,2000

Page 24 of 30

Two perrhenate solutions were used in the characterization of the sensor, a $1 \mathrm{ppm}$ and a $10 \mathrm{ppm}$ solution. An attempt was made during preparation to ensure the only compositional difference between the two was the perrhenate concentration. Additional nitric acid was added to each solution to mimic the acid concentration in the $10 \mathrm{ppm}$ perrhenate solution, and the acid solution added (2.0\% $\pm 0.3 \%$ nitric acid) was a different solution than the perrhenate standards. However, error in the makeup of nitric acid solution should be minimal, with the significant difference between solutions being the perrhenate concentration.

Figure 11 shows the SPR sensor response to successive exposures to perrhenate spiked $\mathrm{NaOH}$ solutions. Initially $4 \mathrm{M} \mathrm{NaOH}$ was pumped through the cell, the pumped stopped, and $1 \mathrm{ppm}$ rhenium in $4 \mathrm{M} \mathrm{NaOH}$ bottle was attached to the input line, and the pump restarted. Flow disruption occurred during each sample change. The pump was also stopped after switching to $10 \mathrm{ppm}$ perrhenate because the flow response was similar to the $1 \mathrm{ppm}$ standard, and we wanted to remove the flow component and check for

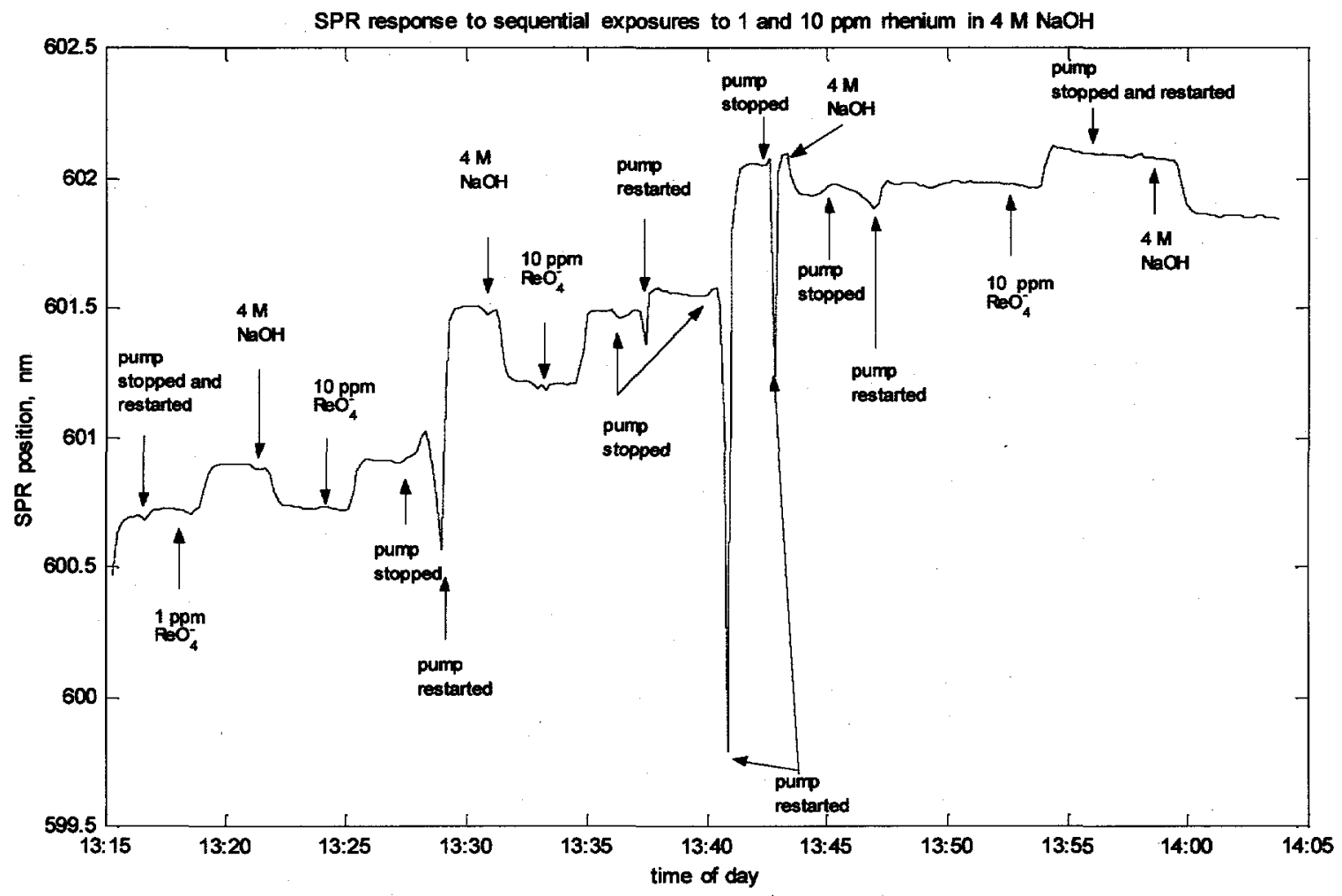

Figure 12. Full sequence of perrhenate exposures shows the difference in response between the blank and the $10 \mathrm{ppm}$ perrhenate solutions. The $\mathrm{NaOH}$ blank response did not return to the $\mathrm{NaOH}$ baseline at the conclusion of the exposures, indicating the presence of additional material sorbed to the SPR sensor surface.

changes in the response. In all cases of pump stoppage in prior experiments, the SPR resonance shifted to lower values immediately upon stoppage. In the case of the $\mathrm{NaOH}$ solutions with no perrhenate present, similar behavior is observed. However, as can be seen in figure 11 , the pump stoppage during the $10 \mathrm{ppm}$ perrhenate sample run resulted in a rise in the SPR position followed by a sharp decrease, and this behavior had not been 
observed for any other solution run to date. The initial rise is indicative of surface adsorption occurring and was not observed for the $\mathrm{NaOH}$ blanks or any of the salt solutions used in calibration. After the initial increase in SPR position, it appears that there is a rapid desorption occurring as evidenced by the sharp decrease in SPR signal. This can be attributed to either hold up in the cell, where liquid of lower refractive index has diffused to the sensor surface or to a temperature increase in the cell.

Figure 12 shows the entire perrhenate exposure run, with every pump stoppage and solution change delineated. Every 10 ppm sample run, except the last, exhibits similar behavior : initial rise in SPR position after the pump was stopped followed by a decline in SPR position until the pump was restarted. This behavior was only observed in the 10 ppm rhenium standard, and is indicative of a rapid and significant rise in solution temperature in the vicinity of the apex of the SPR probe. The fact that the behavior was not seen in the $\mathrm{NaOH}$ blank or in deionized water is another indicator that the sensor is responding to perrhenate in some manner. In addition, the SPR response, after restarting the pump, rose to higher levels than before the pump stoppage for $10 \mathrm{ppm}$ perrhenate, and this is indicative of adsorption of perrhenate onto the surface of the SPR probe, whether by the presence of the PEG adlayer or by direct adsorption onto the metal surface. Finally, the SPR response did not return to the $\mathrm{NaOH}$ blank signal obtained before the perrhenate sample runs, indicating the presence of additional material sorbed to the SPR sensor surface.

An optimistic scenario which explains this behavior is as follows. The perrhenate in solution is passing over the SPR sensor and initially adsorbs to a small degree, as is indicated by the small shift in SPR (this small shift may also be due to a slight refractive index difference between the solutions). The pump is stopped, and this allows perrhenate ion to diffuse to the substrate surface, where is sorbs and initially causes an increase in SPR position. Then, due to the heat of adsorption, the SPR interface heats up locally, causing the SPR position to rapidly decline, as the SPR response is anticorrelated with solution temperature. Restarting the pump causes rapid temperature equilibrium and also allows the SPR response to stabilize at the system temperature, revealing additional material sorbed into the PEG layer (or onto the gold surface). This is repeated several times, until it appears that the surface layer is saturated, and the final perrhenate exposure results in a small shift, and then the response returns to a lower level. The important point here is that over the course of the experiment, the SPR response to $\mathrm{NaOH}$ blank solution does not return to baseline. Rather, the difference between the final and initial $\mathrm{NaOH}$ SPR response is $1.11 \mathrm{~nm}$, indicating the presence of additional adlayer material on the surface of the SPR probe. These results are consistent with SPR response to adsorption of perrhenate ion into or onto the surface of the SPR probe.

It is possible that the system shifted during the experiment, or that lower refractive index liquid was held up in the cell. In the case of the former, the system returned to the baseline response for water after the experiment was concluded, and this indicates that the system did not shift, as any shift which would have yielded a false positive response to perrhenate would also yield a higher water baseline. If lower refractive index liquid was held up in the cell, one would expect the SPR response to dip but then return to the previous value once the pump has started. This behavior was observed in the initial flow 
system characterization where the probe had not been inserted tightly enough. However, the fact that the SPR wavelength position returned to successively higher values after each exposure to perrhenate ion in solution indicates that the sensor responded to successive adsorption of perrhenate to the surface.

\section{Conclusions}

We investigated the feasibility of using surface plasmon resonance as a detection scheme for pertechnetate ion in solution. The literature review indicated that the SPR technique is sensitive enough to detect adsorption of material onto or into a suitable selective film. It was determined that the best method for demonstrating feasibility was to utilize the work performed in the aqueous biphasic chemistry of poly(ethylene glycol) in order to fabricate a sensor for testing.

We fabricated a high performance sensor system which offers the best platform for process in-situ SPR sensing that we have seen in the literature or on the market. This is due to the probe structure and the optical system stability. The sensor was successfully calibrated with respect to refractive index and was successfully used to monitor the formation and degradation of a poly(ethylene glycol) film. The SPR results are consistent with what we observed with spectroscopic ellipsometry. The degradation of the PEG film did not appear to be complete, and the residual PEG left on the surface after rinsing with solvent indicates that the PEG did in fact covalently bond to the gold surface, both in the silicon wafer tests and in the SPR test. Our results are consistent with literature reports of PEGylation of gold films in terms of the attachment phase, and we could not find reports in the literature which approach the long term monitoring of the PEG film that we have performed.

The SPR sensor proved effective in evaluating the formation of the PEG film, and following the final PEG experiment, the sensor was exposed to $4 \mathrm{M} \mathrm{NaOH}$ and ppm levels of perrhenate. The results indicate that material in the perrhenate solution sorbed to the surface and remained on the surface upon additional exposure to $\mathrm{NaOH}$ blank. This is consistent with aqueous biphasic behavior, but the we cannot conclude from these short experiments if in fact the PEG film was present during perrhenate exposure.

We can therefore make the following conclusions: the SPR sensor functions exceptionally well; the attachment of PEG via a thiolate bond may not be as robust as originally expected from literature reports; the sensor responded to perrhenate solutions.

The research performed in this feasibility study points to the utility of SPR for sensing and also validates the SRTC SPR sensor as a high performance SPR platform for remote measurements. The temperature sensitivity associated with the measurements is ubiquitous for all SPR sensor work, and future work should include the integration of a temperature compensation into the probe head. In addition, the flow cell would need to be redesigned in order to direct flow directly onto the apex of the probe and then around the hemisphere, and the cell should be designed with o-ring seals and non-wetting, Teflon walls. These enhancements should remove some of the art associated with placing and 
referencing the SPR sensor. The SPR probe itself needs to be redesigned for stability, and we should work with Equitech to mitigate the rotational asymmetry and etalon effects in the fiber optic coupling to the hemisphere. Finally, a multichannel SPR sensor system will need to be developed in order to investigate differential sensing schemes, where one channel is coated with PEG and the other left as bare gold. The reference channel would serve to remove the effects of temperature and refractive index variations and isolate the surface adsorption response. This approach has some merit as preliminary gold sorption experiments performed after the SPR work was completed indicate that minimal binding of pertechnetate occurs on bare gold surfaces.

The PEG film formation did not proceed as well as expected, and the surface coverages we achieved were significantly lower than we hoped for. The selection of PEG 5000 as the material defined the upper limit, based on literature references found in the course of the research, and we should choose a lower molecular weight PEG for future development. In addition, we did not fabricate a grafted PEG/silica SPR sensor due to time constraints, and it is expected that a grafted PEG would be more robust than the self assembled monolayers formed by PEG thiols and disulfides. The silica substrate would not last long in caustic, and we would therefore need to investigate other grafting routes, where ahumina or titania form the grafting surface.

As stated earlier, poly(ethylene glycol) was chosen as a sorbing material based on the aqueous biphasic extraction work performed by Rogers, and we do not feel this is the best route for sensor development, however, we fell that continued work will be beneficial in the longer term development, i.e. we can work out many of the sensing system technical issues using the non-proprietary PEG surface, and then move onto other, more directly applicable surfaces mentioned in the literature survey section. However, the first task should be to repeat the PEG sorption and perrhenate exposure experiments using the optimized flow cell and sensor configurations as discussed above. The ultimate goal is to develop a sensor with the same functionality as the extraction medium being used in the waste removal process. In conclusion, we feel that all research milestones were met, SPR sensor feasibility has been positively demonstrated, and we are encouraged by the results presented in this report. 


\section{References}

${ }^{1}$ Schmittroth, F.A., de Lorenzo, T.H., Wootan, D.W., Garbick, D.Y., "Inventories for Low-Level Waste Tank Waste", 1995, WHC-SD-WM-RPT-164, Westinghouse Hanford Co.

2 Scwochau, K. "The present Status of Technetium Chemistry", Radiochimica Acta, 1983, 32, 139-152.

${ }^{3}$ Sensors and Actuators. 1999, B54(1-2).

${ }^{4}$ Homola, J.; Yee, S. S.; Gauglitz, G. Sensors and Actuators, 1999, B54(1-2), 16.

${ }^{5}$ Nelson, B. P.; Frutos, A. G.; Brockman, J. M.; Corn, R. M. Analytical Chemistry, 1999, 71, 3928-3934.

${ }^{6}$ Nelson, B. P.; Frutos, A. G.; Brockman, J. M.; Corn, R. M. Analytical Chemistry, 1999, 71, 3935-3940.

${ }^{7}$ Jorgenson, R. C.; Yee, S. S., Sensors and Actuators, 1993, B12, 213.

${ }^{8}$ Jorgenson, R. C.; Yung, C.; Yee, S. S.; Burgess, L. W., Sensors and Actuators, 1993, $B 13-14,721$.

9 Slavík, R.; Homola, J.; Čtyroký, J. Sensors and Actuators, 1999, B54(1-2), 74.

${ }^{10}$ Stemmler, I.; Brecht, A.; Gauglitz, G., Sensors and Actuators, 1999, B54(1-2), 98.

${ }^{11} \mathrm{G}$. H. Cartledge, "The Pertechnetate Ion as an Inhibitor of the Corrosion of Iron and Steel", Corrosion, 1953, 11, 335t-342t.

${ }^{12}$ Cartledge, Groves H. "The Mechanism of the Action of Inorganic Inhibitors", Br. Dent. J. 1966, 1(8), 293-302.

${ }^{13}$ El-Bayoumy, S.; El-Kolaly, M. "Some radiochemical studies on the adsorption behavior of molybdenum- 99 on silver-coated carbon granules and activated carbon.", J. Radioanal. Chem., 1982, 68(1-2), 7-13.

${ }^{14}$ Possibility of using technetium(VII)-99 as a radioactive tracer for monitoring corrosion. Lopukhin, A. P.; Murin, A. N.; Timofeev, S. A. USSR. Radiokhimiya (1988), 30(2), 291-3.

15 Bartsch, R. A., "Metal Complexation by Ionizable Crown Ethers", 1994, DOE/ER/13832-37.

16 Blanchard, D. L., Brown, G. N., Conradson, S. D., Fadef, S. K., Golcar, G. R., Hess, H. J., Klinger, G. S., Kurath, D. E., "Technetium Removal and Characterization: A Progress Report", PNNL, May 30, 1996, Pacific Northwest National Laboratory.

${ }^{17}$ N. C. Schroeder, K. D. Abney, M. Attrep, Jr., S. Radzinski , J. Brewer, K. R. Ashley, J. Ball, F. Stanmore, N. LaFebre, T. Pinkerton, R. Turner, "Technetium Tank Waste 
Partitioning for the Hanford Remediation System: Adsorption and Extraction of Technetium from Double-Shell Slurry Waste Simulant", 1993, LA-U R-93-4092, Los Alamos National Laboratory.

${ }^{18}$ N. C. Schroeder, S.Radzinski, K. R. Ashley, J. Ball, F. Stanmore,G. Whitener "Technetium Partitioning for the Hanford Tank Waste Remediation System: Sorption of Technetium from DSS and DSSF-7 Waste Simulants Using Reillex ${ }^{\mathrm{TM}}$-HPQ Resin", 1995, LA-UR-95-40, Los Alamos National Laboratory.

${ }^{19}$ Rogers, R. D., Bond, A. H., Bauer, C. B., Zhang, J., Rein, S. D., Chomko, R. R., Roden, D. M., "Partitioning Behavior of ${ }^{99} T c$ and ${ }^{129}$ I from simulated Hanford Tank Wastes using Polyethylene-Glycol Based Aqueous Biphasic Systems", 1995, Solvent Extraction and Ion Exchange, 13(4), 689-713.

${ }^{20}$ Rogers, Robin D.; Griffin, Scott T.; Horwitz, E. Philip; Diamond, Herbert "Aqueous biphasic extraction chromatography (ABECTM): Uptake of pertechnetate from simulated Hanford tank wastes", Solvent Extraction and Ion Exchange, 1997, 15(4), 547-562.

${ }^{21}$ Davidson, D.J., Collins, J.L., Anderson, K.K., Chase, C.W., Egan, B.Z., "Removal of Cesium, Technetium, and Strontium from Tank Waste Supernatant.", 1998, ORNL/TM-13612, Oak Ridge National Laboratory.

22 Blanchard, D.L., Brown, G.N., Conradson, S.D., "Technetium in Alkaline, High-Salt, Radioactive Tank Waste Supernate: Preliminary Characterization and Removal", 1997, PNNL-11386, Pacific Northwest National Laboratory.

${ }^{23}$ P.B. Garland, "Optical Evanescent Wave Methods for the Study of Biomolecular Interactions", Q. Rev. Biophys. 1996, 29, 91-117.

${ }^{24}$ P. Pfeifer, U. Aldinger, G. Schwotzer, S. Diekmann, P. Steinrucke, "Real Time Sensing of Specific Molecular binding Using Surface Plasmon Resonance Spectroscopy", Sensors and Actuators B, 1999, 54, 166-175.

${ }^{25}$ The Cauchy model fits the optical constants to the general formula $n(\lambda)=A+B / \lambda^{2}+$ $\mathrm{C} / \lambda^{4}+\ldots$ where $n(\lambda)$ is the refractive index at wavelength $\lambda$, and $A B$ and $C$ are the Cauchy coefficients specific to a given material.

${ }^{26}$ Weast, R.C., Ed., CRC Handbook of Chemistry and Physics, 1989, pg. D-255.

${ }^{27}$ Burns, N. L., Emoto, K., Holmberg, K., Van Alstine, J. M., Harris, J. M. Biomaterials, $19,1998,423-440$.

28 Jung, L. S., Campbell, C. T., Chinowsky, T. M., Mar, M. N., Yee, S. S. Langmuir, 14, 1998, 5636-5648.

${ }^{29} \mathrm{Lu}$, H. B., Campbell, C. T., Castner, D. G. Langmuir, 16, 2000, 1711-1718. 
30 Prime, K. L., Whitesides, G. M. "Adsorption of Proteins onto Surfaces Containing End-Attached Oligo(ethylene oxide): A Model System Using Self-Assembled Monolayers" J. Am. Chem. Soc. 1993, 115, 10714-10721.

31 Palik, I, "Handbook of Optical Constants of Solids", Vol. 1, pg. 293-204.

32 Lee, W. W., Laibinis, P. E., Biomaterial, 19, 1998, 1669-1675.

33 Karlsen, S. "Surface Plasmon Resonance Based Optical Dispersion Sensor", Master's Thesis, 1994.

34 Weast, R.C., Ed., CRC Handbook of Chemistry and Physics, $70^{\text {th }}$ Edition, 1989, pg. E384

35 Jung, L. S., Campbell, C. T., Chinowsky, T. M., Mar, M. N., Yee, S. S. Langmuir, 14, 1998, 5636-5648.

${ }^{36}$ Lu, H. B., Campbell, C. T., Castner, D. G. Langmuir, 16, 2000, 1711-1718 